
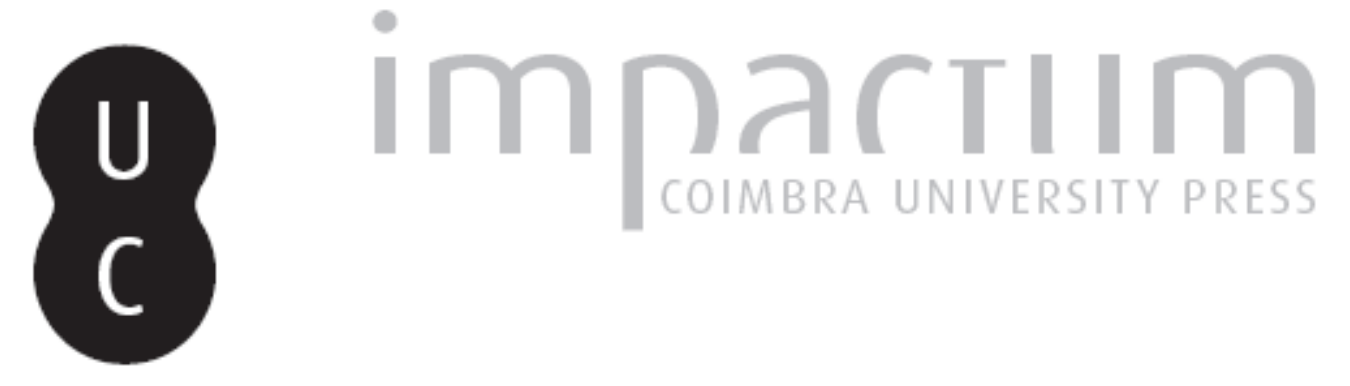

\title{
Images de la culture pombaline dans l'Espagne des Lumières
}

Autor(es): $\quad$ Piwnik, Marie-Hélène

Publicado por: Imprensa da Universidade de Coimbra

URL persistente:

URl:http://hdl.handle.net/10316.2/43918

DOI:

DOI:https://doi.org/10.14195/2183-8925_4-2_15

Accessed : $\quad$ 26-Apr-2023 15:51:39

A navegação consulta e descarregamento dos títulos inseridos nas Bibliotecas Digitais UC Digitalis, UC Pombalina e UC Impactum, pressupõem a aceitação plena e sem reservas dos Termos e Condições de Uso destas Bibliotecas Digitais, disponíveis em https://digitalis.uc.pt/pt-pt/termos.

Conforme exposto nos referidos Termos e Condições de Uso, o descarregamento de títulos de acesso restrito requer uma licença válida de autorização devendo o utilizador aceder ao(s) documento(s) a partir de um endereço de IP da instituição detentora da supramencionada licença.

Ao utilizador é apenas permitido o descarregamento para uso pessoal, pelo que o emprego do(s) título(s) descarregado(s) para outro fim, designadamente comercial, carece de autorização do respetivo autor ou editor da obra.

Na medida em que todas as obras da UC Digitalis se encontram protegidas pelo Código do Direito de Autor e Direitos Conexos e demais legislação aplicável, toda a cópia, parcial ou total, deste documento, nos casos em que é legalmente admitida, deverá conter ou fazer-se acompanhar por este aviso.

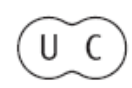




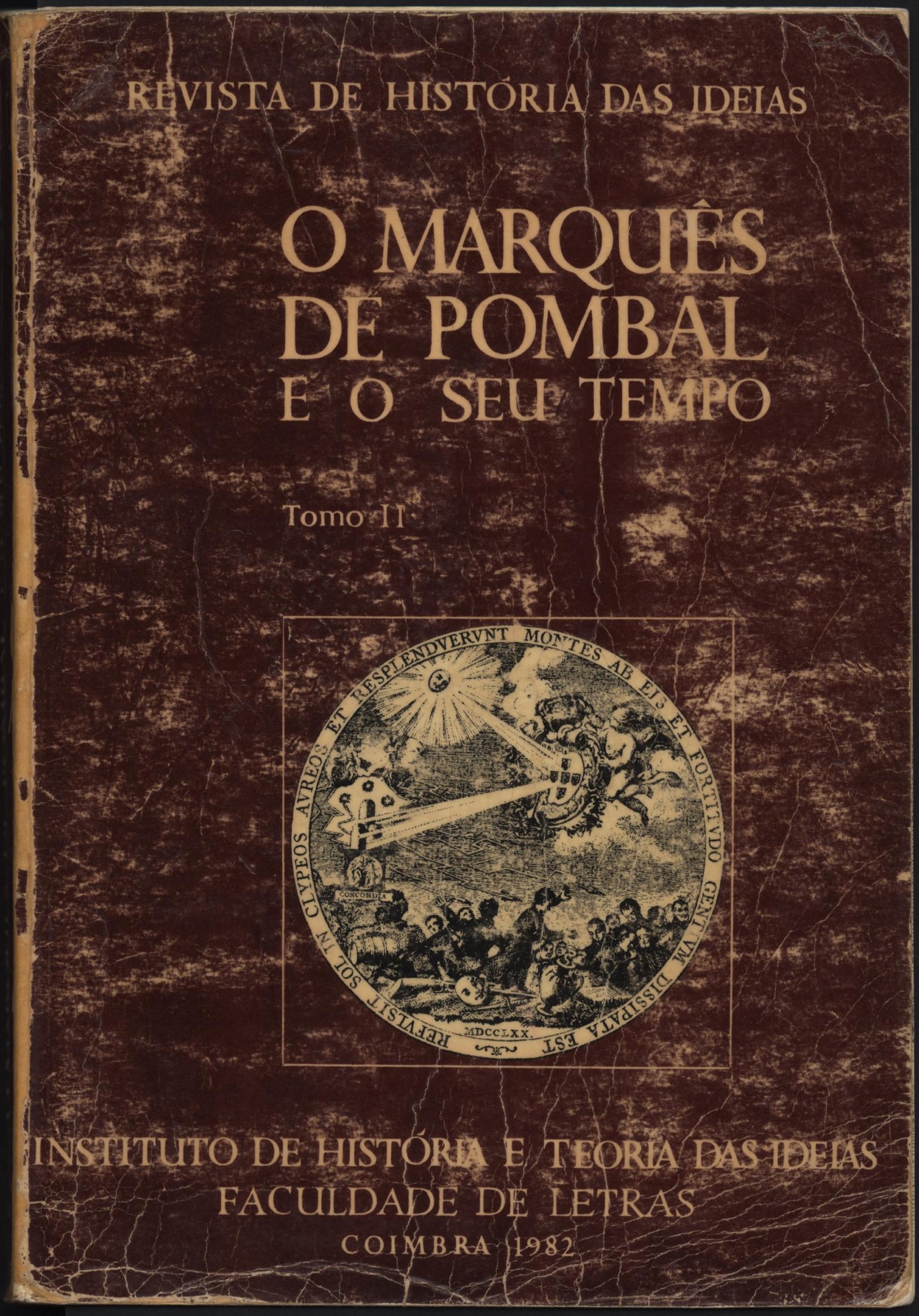




\section{MARIE-HELENE PIWNIK *}

\section{IMAGES DE LA CULTURE POMBALINE DANS L'ESPAGNE DES LUMIERES}

La Gaceta de Madrid, à l'instar d'autres périodiques européens du même genre, outre qu'elle communiquait au public les nouvelles de la semaine pays par pays, comportait à la fin de presque tous ses numéros des petites annonces consacrées dans leur majeure partie aux ouvrages récemment parus en Espagne, avec toutes les indications nécessaires sur l'auteur, le titre, parfois le contenu, le lieu et la maison d'édition, ainsi que les points de vente à Madrid et dans le reste de la Péninsule $\left(^{1}\right)$. Il s'agit donc d'une source d'informations précieuse qui donne un accès direct à l'une des manifestations culturelles les plus importantes au XVIII ${ }^{e}$ siècle, la librairie, en essor constant grâce au développement de la typographie, des relations postales et du niveau intellectuel d'une partie de la population.

Nombre des oeuvres annoncées sont des traductions déclarées comme telles - certaines peuvent l'être sans en faire état - , et le poids de cet apport a pu être mesuré, qui fait peser la balance en faveur des ouvrages français, puis italiens et portugais, les autres langues arrivant loin derrière $\left({ }^{2}\right)$. J'ai été conduite à ce sujet, et à propos d'ouvrages de Teodoro de Almeida mis en souscription auprès des Espagnols, à poser diverses questions qui ouvrent un débat plus qu'ils ne le résolvent, concernant la réception par un public donné de textes qui lui

* Universidade de Paris $\mathrm{x}$ - Nanterre.

(1) P.-J. Guinard, La presse espagnole de 1737 à 1791, Formation et signification d'un genre, Centre de Recherches Hispaniques, Paris, 1973,572 p.. Sur la Gaceta de Madrid plus précisément, voir l'index.

$\left(^{2}\right)$ François Lopez, Juan Pablo Forner et la crise de la conscience espagnole au XVIII. siècle, Université de Lille III, 1977, p., en particulier pp. 64-80, 473-480. 
parviennent en tout état de cause dans sa langue et, lorsqu'il s'agit de la péninsule, largement auto-censurées par le traducteur lui-même avant d'être soumises de toute manière aux censeurs officiels $\left({ }^{8}\right)$.

On s'aperçoit en effet que la traduction a rarement pour objectif de dépayser son lecteur ou de véhiculer des connaissances sur le pays rédacteur ou son esthétique littéraire, et cherche plutôt à conditionner un public ou à répondre à sa demande, à l'aide de modèles didactiques pris là où ils ont déjà fait la preuve de leur efficacité, y compris lucrative. En ce sens, on pourrait aller jusqu'à dire que la France, qui arrive en tête des traductions, est le pays dont l'idéologie correspond le plus aux besoins de l'élite éclairée espagnole, mais à la suite d'un choix qui écarte ou ampute le plus souvent les ouvrages profondément rénovateurs au bénéfice de romans à l'eau de rose ou de livres pieux.

Ainsi les pays dont on traduit les publications sont en quelque sorte des viviers où l'on puise, plus qu'ils ne sont des foyers qui irradieraient d'eux-mêmes. Publications que l'on adapte aux exigences du pays d'accueil, plus soucieux de l'utilité nationale et des chances de succès que de l'exactitude de la transmission, ce qui explique d'ailleurs les emprunts inavoués avec hispanisation des noms propres étrangers et autres subterfuges.

Le Portugal dans cette perspective occupe une place flatteuse dans les colonnes des petites annonces de la Gaceta de Madrid, que la proximité géographique ne suffit pas à expliquer, et à laquelle aurait pu faire obstacle un contentieux historique qui lui donne en 1640 son indépendance définitive au détriment de son hégémonique voisine. Les facteurs qui font de ce pays dans une certaine mesure un exemple structurel sont trop connus pour qu'on y insiste, car le tremblement de terre de 1755 et l'ascension de Pombal impliquent des répercussions dans toute l'Europe, ne serait-ce que par l'expulsion des Jésuites. Afin d'apprécier à sa juste valeur l'intérêt qu'il suscite en Espagne, j'ai donc entrepris un recensement des traductions d'ouvrages portugais en espagnol proposées aux lecteurs de la Gaceta à partir de 1750. Ce dépouillement exhaustif, travail fastidieux que les chercheurs mènent encore seuls, faute de moyens pour constituer des équipes et surtout informatiser et diffuser les résultats, n'est cependant pas une tâche insur-

( $\left.{ }^{3}\right)$ Marie-Hélène Piwnik, «Les souscripteurs espagnols du P. Teodoro de Almeida», Bulletin des études portugaises et brésiliennes, T. 42, 1981 . 
montable, et il parait de prime abord aisé d'en produire les conclusions, s'il suffit d'énumérer, par exemple dans l'ordre chronologique, les ouvrages qui apparaissent traduits.

Mais le découpage de la période introduit une problématique que j'esquisserai avant tout autre chose. Le Bi-centenaire de la mort du Marquis de Pombal m'a en effet engagée à examiner la diffusion de la culture portugaise en Espagne - dans les termes restrictifs que j'ai posés plus haut - , au moment où Sebastião de Carvalho exerce le pouvoir: ses actions à l'endroit de la censure antérieure, avec en particulier la création de la Real Mesa Censória, ses efforts en faveur de l'impression avec la fondation de la Regia Officina Typographica, illustrent en effet de façon significative l'apogée des Lumières au Portugal. Or, il se trouve que certains titres, publiés dans ce pays sous Pombal ne sont traduits en Espagne qu'à la fin du siècle. Ils véhiculent cependant indéniablement, dans une optique portugaise, une image de la culture pombaline. Mais on peut douter qu'elle soit retenue en ces termes par le public espagnol dix ou quinze ans après la chute du favori de José I.

A l'inverse, le Verdadeiro Método de estudar, qui paraît au Portugal bien avant l'ascension du Comte d'Oeiras, n'est traduit en Espagne qu'après le tremblement de terre, et l'on peut être sûr qu'il est ressenti par les lecteurs espagnols comme un authentique symbole de toute la réforme doctrinale que l'on verra entreprise et appliquée par le régime pombalin, en particulier à Coimbra. On voit donc qu'il convient d'envisager une période beaucoup plus large qu'il n'y paraît, pour appréhender avec une fiabilité d'ailleurs toute relative, le rayonnement culturel d'une époque dont les limites chronologiques sont pourtant clairement établies: 1750-1776. J'ai ainsi tenu compte des oeuvres publiées au Portugal entre ces deux dates, même lorsqu'elles étaient annoncées dans la Gaceta plus tard dans le siècle; et aussi des oeuvres annoncées en Espagne pendant cette période, même lorqu'elles étaient antérieures.

Il convient aussi de dire que l'enquête menée ne donne qu'une idée très partielle de la diffusion de la culture portugaise en Espagne dans la deuxième moitié du XVIII ${ }^{\mathrm{e}}$ siècle, qu'il y aurait lieu de compléter par le dépouillement, par exemple, des articles du Memorial Literario $\left({ }^{4}\right)$, par les catalogues

(') Cette publication, qui commence en avril 1784 (numéro de janvier de la même année) donnait à ses lecteurs des nouvelles de la vie littéraire: livres nouveaux, travaux des Académies; il leur proposait aussi des compte-rendus des spectacles, et les tenait au courant de l'actualité scientifique. P.-J. Guinard, op. cit., pp. 252-265. 


\section{O Marquês de Pombal}

de libraires, le relevé de la Biblioteca periódica anual $\left({ }^{5}\right)$, les correspondances $\left(^{6}\right)$, etc. On n'oubliera pas qu'à côté des traductions circulent en Espagne, au sein de l'élite, des ouvrages en version originale portugaise, et l'on signalera enfin que certains titres d'importance, comme la Tentativa theologica, d'António Pereira de Figueiredo, ne reçoivent pas les autorisations de parution nécessaires.

Le problème des traducteurs, souvent galiciens, ne sera pas abordé dans ce court article, mais il est clair que pour beaucoup d'entre eux, tels Benito Bails $\left({ }^{7}\right)$, ou Maymó y Ribes $\left({ }^{8}\right)$, la publication d'ouvrages célèbres à l'étranger est à la fois une source de bénéfices et un tremplin pour la mise en vente de leurs propres écrits et le lancement de leur carrière littéraire ou scientifique.

$\left({ }^{5}\right)$ Les imprimeurs donnent à la fin des ouvrages qu'ils publient des listes de livres en vente chez eux ou dans les librairies qui diffusent leurs productions. Il y avait aussi des catalogues séparés. Cf. A. Rodríguez-Moñino, Historia de los catálogos de librería española (1661-1840), Madrid, 1966. Sur la Biblioteca periódica anual, v. F. Lopez, op. cit., p. 474.

(') La correspondance de Manuel do Cenáculo avec divers Espagnols parmi les plus illustres, entre autres Gregorio Mayáns, manifeste avec éclat cette circulation de librairie, dont on peut déjà se faire une première idée en consultant le catalogue de la Bibliothèque d'Evora mis au point par Nobre de Gusmão.

(') Benito Bails fut Directeur d'Etudes Mathématiques à la Royale Académie des Beaux-Arts de San Fernando, membre des Académies de la Langue et d'Histoire, de l'Académie des Sciences Naturelles et Arts de Barcelone. Diverses de ses oeuvres sont annoncées dans la Gaceta de Madrid après qu'il s'est fait connaître par sa traduction de Ribeiro Sanches. Ainsi dans le n. 17 de 1786, Pruebas de ser contrario a la practica de todas las naciones y a la disciplina eclesiástica, y perjudicial a la salud de los vivos, enterrar los difuntos en las iglesias y los poblados, Madrid, Ibarra, 1785; dans le n. 9 de 1788, Lecciones de clave $y$ principios de harmonia, Madrid, V.da de Ibarra, reed. de la publication de 1776, dont Palau dit qu'il s'agit d'une traduction de Antonio Bemetz-rieder. En 1790, deux ouvrages, Aritmética para negociantes, Madrid, V.da de Ibarra, et Elementos de matemática de la Real Academia de San Fernando, imprimés en 1779 par Ibarra.

$\left.{ }^{(}\right)$Maymó y Ribes, peu après avoir traduit le Verdadeiro Método et s'être engagé dans la polémique que l'on sait avec le P. Isla, publiait «un livre nouveau», Proemiales de la Jurisprudencia, «su origen y progresos, y Comentarios à las leyes del Código Papyriano, y XII Tablas Obra muy curiosa, y util a todos» annoncé dans la Gaceta de Madrid n. ${ }^{\circ} 17$ de 1767. Le livre est en vente «avec l'oeuvre du Barbadiño et sa Défense», chez Francisco Fernández. En 1768 (n. 28) la référence à Vernei se maintient. En 1770, les Proemiales sont proposés seuls au public, ce qui tend à prouver que la popularité de Maymó est désormais suffisante pour évacuer un parrainage prestigieux mais encombrant. Après la mort de Maymó la Gaceta n..$^{\circ} 9$ de 1788 offrira encore à ses lecteurs son livre Romani et Hispani Juris Institutiones ad usum scholae et fori. Il a sa clientèle... 
La première constatation qui s'impose en considérant le recensement effectué, c'est que rien de ce qui touche la poésie ou le théâtre portugais ne franchit les frontières espagnoles, sous forme de traduction en tout cas. Je ne m'attarderai pas sur ce point, peut-être en rapport avec la décadence au Portugal de l'influence espagnole au profit d'un courant français de culture donnant lieu à l'Arcádia et au théâtre de Manuel de Figueiredo déjà en début de siècle, alors qu'en Espagne la régression du théâtre que l'on appelle postcaldéronien et l'apparition de modèles nouveaux sur la scène ou dans les formes poétiques attend en sommela dernière décade. Il est difficile toutefois d'en inférer que le public espagnol n'est pas susceptible de s'intéresser à la traduction de pièces néo-classiques portugaises et de poèmes arcadiens à l'époque qui nous occupe, car le théâtre français et la poésie de Voltaire, par exemple, sont traduits. Il est plus raisonnable de penser que pour ces deux genres l'exemple vient essentiellement de France et que les traducteurs se tournent vers ce pays lorsqu'ils éprouvent le besoin d'offrir à leur public des images littéraires qui en soient les applications les plus réussies.

Quoi qu'il en soit, c'est dans le domaine de la rénovation de la pensée, philosophie, médecine, religion, que se recrute une bonne partie des traductions de portugais en espagnol, et l'on peut affirmer que beaucoup des grands noms de l'Iluminismo sont présents dans les petites annonces de la Gaceta de Madrid. Mais il faut dire d'emblée qu'ils alternent avec ceux d'illutres inconnus, ou de célébrités oubliées du siècle antérieur. Dans cette mesure, un classement par matières s'avère peu pertinent, ne rendant compte que des oeuvres elles-mêmes, saisies dans leur illusoire éternité, et non de l'attente du pu. blic $\left({ }^{9}\right)$ ni des exizences du pouvoir.

On ne saurait, bien entendu, négliger cependant la distinction Sciences, Lettres, Morale, etc., sous peine de confusion, mais elle autorise de nouveaux regroupements, qu'il m'a semblé plus juste et plus efficace d'établir à partir de trois courants-force, très représentatifs d'ailleurs de l'esprit éclairé: ouvrages, laïcs ou religieux, que réunissent un même esprit, un même effort didactique inspiré par la raison; ouvrages qui flat. tent l'éveil de la sensibilité, voire de la sensiblerie, chez un public peut-être épris d'irrationnel par contre-coup; ouvrages suscités par la circonstance politique et qui propagent temporairement et au moment opportun l'idéologie gouvernementale.

$\left(^{\circ}\right)$ Sur ce point, Hans Robert Jauss, Pour une esthétique de la réception, Gallimard, Bibliothèque des Idées, Paris, 1978, $305 \mathrm{p}$. 
Les travaux scientifiques portugais occupent une place d'honneur dans les petites annonces de la Gaceta de Madrid, cristallisées en début et fin de période respectivement par Vernei et Teodoro de Almeida.

C'est dans le n. 22 de 1760 qu'apparait le Verdadero Método de estudiar para ser util a la Republica, y a la Iglesia: «su Autor el R. P. Barbadiño de la Congregacion de Italia: traducido del Idioma Portuguès al Castellano por el Doct. Joseph Maymo y Ribes, Abogado de los Reales Consejos, y del Colegio de esta Corte: Trata el Autor en esta Obra de todas las Facultades, mayores, y menores, de Matematica, Cronologia, Historia antigua, y moderna, lenguas orientales, Griega, y Hebréa, Retoria, Politica, y Poesia; manifiesta los abusos, que se han introducido en estos estudios, propone lo que se debe aprender en cada uno de ellos, su metodo, y Libros de que se debe usar para su reforma. Son quatro Tomos, a que se seguiran las Apologias, y Defensas del Autor; se venden en casa de Marcos Ruiz de Tejada, Mercadèr en los Portales de calle Mayor, frente la casa de la Estera». Dans le $n^{\circ} 13$ de 1761, la mise en vente de l'ouvrage à Séville, chez Rafael Cubillo, est annoncée, puis en $1763\left(n^{\circ} 12\right)$, apparaît le nom d'un deuxième libraire à Madrid, Francisco Fernandez, calle de las Carretas. En 1768 ( n $^{\circ} 9$ ), sont offertes au public les "Apologias del R. P. Arsenio de la Piedad, y de D. Aletofilo Cándido de la Cerda, á la obra del R. P. Barbadiño, intitulada Verdadero Método de estudiar, y Respuestas á ella dadas», par le même traducteur. S'y joignait la Defensa del Traductor, riposte de Maymó y Ribes aux critiques acerbes du $P^{e}$ Isla dans Fray Gerundio de Campazas $\left({ }^{10}\right)$.

L'ouvrage de Vernei parut au Portugal en 1746 et attendit donc quatorze ans avant de paraître en Espagne à la suite de problèmes de censure que divers documents conservés dans les Archives Nationales de Simancas et de Madrid permettent d'apprécier dans une grande mesure.

C'est en effet dès mai 1755 que le tome I de l'ouvrage en version espagnole est remis à Juan Curiel, fiscal du Conseil de Castille, avec une demande de licence pour dix ans ( ${ }^{11}$ ). Celui-ci transmet au censeur D. Pedro José Pérez Valiente, «del Consejo de Su Majestad y Theniente de Corregidor de la Corte», qui,

${ }^{(10)}$ Pour toute cette question, v. António Alberto Banha de Andrade, «Bibliografia da polémica verneiana», Brotéria, vol. XLIX, Agosto-Setembro 1949. Et «Edições clandestinas do Verdadeiro Método de Estudar e folhetos da polémica, Filosofia, Ano VIII, 1961. 50651 .

(11) A.H.N.M. (Madrid) Consejos, fndice de Impresiones, Leg ${ }^{\circ}$ 
selon la formule consacrée, n'y ayant rien trouvé de contraire à la religion et à l'Etat, en autorise la parution le 15 mai 1755 , la licence et le privilège de dix ans étant passés le 2 juin $1755{ }^{\left({ }^{12}\right)}$. Le 8 juin 1757, le censeur Isidoro Rubio donne son avis sur le tome II, qu'il trouve digne d'impression à l'exception d'un passage contenant des

«proposiciones impertinentes, falsas, turbativas de la paz; y denigrativas de un sujeto de la primera fama en la República de las letras y a quien Su Majestad, Dios le guarde, ha honrado en su persona y escritos»

- il s'agit du morceau bien connu sur Feijoo - $\left({ }^{13}\right)$. La licence est accordée le $\mathrm{I}^{\text {er }}$ juillet $1757\left({ }^{14}\right)$. A peine deux mois plus tard, ordre est donné de suspendre l'impression chez Ibarra, de saisir les originaux et de détruire les moules $\left({ }^{15}\right)$. C'est le marquis del Campo de Villar qui transmet le 19 septembre à Curiel la royale décision, due à l'intervention du Procureur Général des Jésuites de la Province de Tolède, Francisco Lozano, auteur d'une longue dénonciation circonstanciée, conservée à Simancas, non datée, et précédé d'une lettre également non datée $\left({ }^{18}\right)$ qui résume les positions de son auteur à propos du Verdadeiro Método:

«una de las mas disimuladas Satyras contra la Nacion Portuguesa, y contra la Comp. ${ }^{2}$ de Jhs, y como tal há sido prohibida en aquel Reyno, con las mas rigurosas penas; por denigrar sobre manera à todas sus Academias, y Escuelas, satyrizando las Ciencias sagradas, y profanas, $\mathrm{q}^{\mathrm{e}}$ se cultivan en el (...) obra (...) por consiguiente tan deshonorativa de todos los estudios, sagrados y profanos de las Universidades de España, en donde se cultivan con el mismo methodo todas las expresadas Facultades»

Et d'ajouter qu'il faut «éteindre les étincelles de désunion, altercations, disputes et écrits satiriques en tous genres que ce libelle ne manquera pas de faire surgir».

La dénonciation, elle, est divisée en trois parties:

1. Rubriques satiriques de l'oeuvre anonyme publiée par le pré tendu Barbadiño.

\footnotetext{
(12) Ibid.

(18) Ibid., Leg ${ }^{\circ} 50653$.

('4) Ibid.

( $\left.{ }^{28}\right)$ A.G.S. (Simancas), Gracia y Justicia, Leg 979.

(16) Ibid. et A.H.N.M., Consejos, Comisión de Imprentas, Leg. 0 s 51633-51634.
} 
2. Quelques-unes des propositions, parmi beaucoup d'autres, contenues dans les deux tomes, et dignes de censure.

3. Propositions dignes de grave censure, tirées de la riposte donnée aux Réflexions de Fr. Arsenio de la Piedad (Valence, Antonio Valle, 1748).

L'argumentation développée sommairement dans la première partie stigmatise à partir d'un certain nombre de lettres l'anti-nationalisme de Vernei et s'élève en particulier contre la satire de Vieira.

Les citations choisies dans la deuxième partie soulignent l'animosité de l'Archidiacre à l'égard de la Compagnie et de son fondateur, et son indulgence pour Luther et Calvin. Anti-jésuitisme et hérésie sont donc les deux accusations portées au Verdadeiro Método.

Enfim dans la troisième partie sont citées une dizaine de passages répréhensibles, entre autres celui qui condamne Aristote et les commentaires de Saint-Thomas, et celui qui donne la priorité à Grotius sur tous les jurisconsultes portugais.

L'ensemble est extrêmement faible et d'une veine obscurantiste indéniable.

Le 20 septembre, Juan Curiel faisait savoir au marquis del Campo qu'il avait fait exécuter l'ordre royal, ne laissant imprimer que quatre exemplaires à l'endroit des censeurs qui seraient nouvellement désignés. Mais il tient à donner son point de vue personnel sur l'oeuvre du Barbadiño:

«Yo siempre he tenido por muy util esta obra, de mucha y provechosa instruccion, sin adherir á sus systemas, ni hazer detenida refleccion en los particulares que ha notado el $\mathrm{P}^{\mathrm{e}}$ Lozano que en la mayor parte son de aquellos que pudieran ponerse à muchas de las obras que corren impresas: pero estoy persuadido á que há de dar mucho fruto, pues da luz, y propone medios para lo mejor, notando, y advirtiendo lo que necesita de enmienda, haciendo manifiestos los vicios y los defectos, para que afeados se abstengan de ellos los culpados, y aunque no remedie otra cosa que el que oigamos la palabra de Dios en los pulpitos como palabra de $\mathrm{D}^{\mathrm{s}}$ y no como fantasia de los hombres y agudezas de entendimiento abremos adelantado mucho» $\left({ }^{17}\right)$.

(") A.G.S., Gr. y Just., Leg 979. 
De son côté Maymó y Ribes rédigeait une Breve demonstracion de la profunda, y sana doctrina del Barbadiño, utilidad, y necesidad de su Obra titulada Verdadero Método de estudiar, para ser util à la Republica, y a la Iglesia, du 3 novembre $\left.1757{ }^{18}\right)$. Après avoir établi une nette distinction entre satire et critique, il a recours à l'histoire pour montrer l'utilité d'une réforme des méthodes d'enseignement, et se félicite qu'elle soit à l'ordre du jour dans la Péninsule:

"En Portugal, por haver admitido Juan V el metodo de los extrangeros. logrò mejorar en Architectura, Musica, Fabricas de Navios, polvora, seda, vidrios etc. introdujo à modo de Paris una Academia de Historia. En España tenemos modernam ${ }^{\text {te }}$ fundadas muchas $\mathbf{R}^{\text {es }}$ Academias, Fabricas, Catedras de Lenguas Orientales en las Universidades, no por otra razon, sino porque el mundo va de cada dia abriendo mas los ojos, adelantando à los antiguos, y emendando sus defectos».

Puis il passe en revue toutes les lettres du Verdadeiro Método pour en dégager l'intérêt, affirmant que jamais aucun Portugais ni Espagnol n'est mis en cause de façon injurieuse. Ainsi le propos sur Vieira est très nuancé, et un hommage senti lui est rendu en maints endroits. Si le Bernardiño critique certains défauts des Comédies espagnoles, il le fait avec de si bonnes raisons qu'il convainc le plus hésitant. Quant à Aristote, c'est la façon dont on l'enseigne qui est "embrouillée et sans aucune utilité ". C'est d'ailleurs ce que pense $\mathbf{P}^{\mathbf{e}}$ Herreros qui vient de publier un cours de Philosophie moderne. En ce sens la Physique nouvelle n'a pas à être défendue, elle donne des preuves éclatantes de ses mérites en Espagne même, comme le signale l'ouvrage portugais en évoquant les Académies de Physique Expérimentale et Médecine de Séville et Madrid. Enfin, conclut Maymó:

"Queda demonstrado, que la doctrina del Barbadiño en todas sus cartas se conforma con lo que han dicho, y escrito los mejores Jesuitas».

Les Oratoriens ont été vilipendés pour avoir voulu introduire des méthodes semblables, mais aujourd'hui on les applique avec profit. Il en ira de même pour le Verdadeiro Método.

(18) Ibid. 
Et il accompagne sa démonstration d'une lettre, s. d., probablement à Curiel, où il le prie de choisir avec soin les nouveaux censeurs:

«se ha de servir V. S. no remitirla a ningun Individuo de las Escuelas Dominicana, Jesuitica, y Franciscana, pues estos doctos Religiosos tienen hecho voto de seguir el metodo y opiniones de sus escuelas, y reprobaràn qualquiera reforma util de muchas, que nota el Barbadiño» ${ }^{(19)}$.

Furent désignés le $\mathrm{P}^{\mathrm{e}} \mathrm{D}^{\mathrm{n}}$ Juan de Aravaca, de l'Oratoire du Salvador, et le $\mathbf{P}^{e} \mathrm{D}^{\mathrm{n}}$ Miguel Alvira, de l'Oratoire de San Felipe Neri. On peut avoir une idée assez précise de leurs censures par un rapport très complet mis au point par Curiel pour le marquis del Campo le 2 février $1758\left({ }^{20}\right)$. Il s'agit d'une épaisse liasse de 31 pages, qui rappelle les diverses étapes de «l'affaire», avant de souligner que les censeurs choisis ne sont pas concernés par les méthodes d'étude et les écoles critiqués par le Barbadiño, et offrent donc toutes garanties d'objectivité. Ils se sont prononcés sur le Verdadero Método, sur la dénonciation de Lozano, sur la Démonstration de Maymó et sur la Riposte à Fr. Arsenio de la Piedad. Tous deux ont déclaré que non seulement il n'y avait aucune raison pour qu'on ne continuât pas l'impression de l'ouvrage, mais qu'il devait être considéré comme très utile pour l'Espagne, et que son traducteur devait être félioité et récompensé pour son zèle et le bien-fondé de son entreprise destinée à répandre de saines doctrines dans la nation. Curiel pour sa part est persuadé que des motif condamnables ont poussé Lozano, qui ne pouvait ignorer que le Conseil de Castille lui-même avait accordé la licence, en parfaite connaissance de cause. N'était-ce pas faire injure à Sa Majesté, d'ailleurs, que de penser qu'elle n'était pas au courant d'un livre connu de toute l'Europe, comme d'ailleurs les pamphlets qu'il a entraînés depuis dix ans, tant au Portugal au'en Espagne, et que le Jésuite se contente tout simplement de reprendre? Fallait-il l'importuner quand des affaires plus urgentes la retiennent, et ceci ne signifie-t-il pas que Lozano avait échoué auprès de l'Inquisition elle-même?

Quoi qu'il en soit, Aravaca et Alvira ont confirmé la décision des premiers censeurs concernant le passage relatif à Feijoo: il est donc définitivement supprimé. Pour le reste, Ara-

\footnotetext{
('9) Ibid.

( ${ }^{(0)}$ A.H.N.M., Consejos, Comisión de Imprentas, Leg. ${ }^{\circ}$ 51633-51634 .
} 
vaca a également donné satisfaction à Lozano, dans une certaine mesure, pour ses remarques à propos de Saint-Ignace. Le morceau sera légèrement modifié. Mais Lozano aurait pu censurer la doctrine du Barbadiño concernant la venue de l'apôtre Saint-Jacques en Espagne, que le $\mathrm{P}^{\mathrm{e}}$ Alvira considère irrecevable:

«y era muy propio del celo de nuestra nacion no permitir que en estos Reynos se declaren opiniones contrarias a ñtra piadosa tradicion»

De toutes façons, conclut Curiel:

«no puede embarazarse la ediccion de esta obra por la grande utilidad que ha de traer al publico, vorrense, ô no so vorren, notense, ô no se noten los pasajes referidos»

La Nation y trouvera des bases pour l'établissement de ses méthodes d'étude, saura choisir ce qu'il y a de mieux, et laisser de côté ce qui ne convient pas.

En marge des premières pages de ce document, quelques paragraphes signés du marquis del Campo autorisent en date du 5 janvier 1760 la continuation de l'impression, par décision royale $\left({ }^{21}\right)$.

Entre temps, il avait fait tenir à Carlos III tout le dossier, avec une lettre personnelle qui reprend plus ou moins les termes du rapport de Curiel, et donne d'autres indications sur l'opinion exprimée par les censeurs:

«Uno y otro (...) expresan que esta obra publicada en Portugues quince años ha, es buscada y leida por todos los curiosos de la Europa, y que la oposicion de los que han solicitado destruirla, no ha logrado otra cosa que manifestar su malignidad, y afianzar mas la excelencia y utilidad de ella, la que de muchos años a esta parte se vende en España, y de imprimir aora en Castellano, solo puede seguirse la maior facilidad que tendrán para leerla los que no entienden bien el Portugues» $\left({ }^{22}\right)$.
(21) Ibid.
(22) A.G.S., Gr. y Just., Leg 979. 
La satire, poursuivent-ils, concerne les moeurs et ridiculise les faiblesses et vices des hommes; la critique ne vise pas les personnes, mais les manifestations de l'entendement afin d'en corriger les erreurs pour établir une science autorisée et utile. En Espagne les vrais sages, persuadés de la nécessité d'une réforme des études, travaillent en ce sens:

«como lo muestra en su curso philosophico el $\mathrm{P}^{c}$ Luis Losada, el que và publicando el Maestro Fr. Manuel de Rivera, las Obras del Dr. Piquer, las Conclusiones Philologicas que se defienden en Valencia, Cerbera, y Seminario de Nobles de esta Corte, con otros escritos que en el dia componen algunos doctos Jesuitas».

A Rome, le Pape a ordonné que l'on change entièrement le système des études pratiqué au siècle dernier, et exilé Aristote, créant

"Cathedras de Theologia Dogmatica, Historia Eclesiastica, Instituciones Canonicas y Civiles, Medicina Theorica y Practica, Cirurgia, Anathomia, Chimica, Phisica Newtoniana, Logica, Metaphisica Eclesiastica ò libre y de lenguas Griega, Hebrea, Siriaca, Ara. biga, y otras orientales, nombrando Lectores del Clero secular, y de todas las religiones, sin admitir sistemas particulares de escuelas, cuio metodo siguen en Bolonia, Padua, Napoles, Turin, Viena, Paris, y casi lo restante de Europa, y ès el sistema que propone el Barbadinho, de que se evidencia la necessidad que hay de reformar el nuestro, que ha cerca de dos siglos se dejò en otras partes, de que resulta el desprecio con que nos tratan las demas Naciones, reputandonos por ignorantes y presumidos, diciendo en sus libros que España es un Pais incognito para los savios, aue no tienen comercio literario con el resto de la Europa, ni hay en ella hombres doctos que sepan perfectamente las Ciencias».

Ainsi l'ouvrage de Vernei permettra à l'Espagne de rejoindre le concert des nations européennes éclairées $\left({ }^{23}\right)$.

Le Roi mit pourtant presque deux ans à donner son accord, et l'on peut imaginer les pressions qui s'exercèrent pour

(2) Ibid. 
l'en empêcher, dont assurément celle de sa femme, choquée par les critiques adressées à son pays d'origine, comme le raconte. plaisamment le $\mathrm{P}^{\circ}$ Isla à l'un de ses correspondants, arguant que la Reine s'est opposée à la publication de la traduction parce que l'auteur disait "trop de mal des Portugais" $\left({ }^{24}\right)$.

De Vernei est annoncé également, dans la Gaceta $n^{\circ} 20$ de 1769 le De Re Logica, ad usum lusitanorum adolescentium, Libri sex, (1751), que je ne mentionne que pour mémoire, car il s'agit de la réédition en version originale - en latin - de Valence, mise au point par Juan Bautista Muñoz.

Si l'on s'en tient aux traductions, le deuxième grand pôle d'attraction dans le domaine des Sciences, et qui équilibre l'astre Vernei est, bien entendu Teodoro de Almeida, avec la Recreación Filosófica, qui parut à Lisbonne de 1751 à 1768, mais ne fut proposée au public madrilène qu'en 1787 ( $\left.n .^{\circ} 45\right)$, en ces termes:

«es un tratado completo de Física o Filosofía natural con la particular ventaja sobre otros que se han escrito en este género, de que se explican en él todas las materias de esta ciencia, sin omitirse nada de quanto han adelantado los modernos en sistemas, experiencias, observaciones y máquinas, de un modo tan sencillo y claro que lo puede entender quien no sepa la Matemática».

Le censeur espagnol chargé de rapporter sur l'oeuvrè, Francísco Messeguer y Arrufat, se montre extrêmement favorable:

«De tantas obras como se traducen en estos tiempos, pocas o ninguna seràn tan utiles como esta Recreacion Filosofica del P. Almeida (...). Porq ${ }^{e}$ de nada està mas escaso ñro idioma y nada mas necessitamos que buenos tratados de Fisica ò de Filosofia natural en lengua castellana para $\mathrm{q}^{\mathrm{e}}$ pueda de algun modo instruirse la Nacion en la buena filosofia mientras no se enseñe en las escuelas publicas» $\left({ }^{25}\right)$.

C'est, poursuit-il, un traité très complet, qui comprend les principales parties de la Physique: l'homme, les ani-

\footnotetext{
(24) P. José Francisco Isla, Obras escogidas, B.A.E., T. XV, p. 479.

${ }^{25}$ A.H.N.M. Consejos, fndice de Impresiones, Leg. ${ }^{\circ}$ 5551-5.
} 
maux, les éléments, la lumière et les couleurs, le Ciel et les Planètes; et qui a l'avantage d'être très accessible:

«de todo esto trata con el mejor orden, valiendose de las ultimas observaciones y experiencias, y sobre todo con tanta claridad que le puede entender qualquiera que tenga una mediana razon aunq no haia frequentado las aulas; siendo este el unico fin $\mathrm{q}^{\mathrm{e}}$ dice el mismo Autor que se propuson.

On a voulu le traduire à plusieurs reprises en Espagne, mais on y a renoncé à chaque fois, «car il n'est pas si facile pour nous qu'il y paraît de faire une bonne traduction du portugais». Raison de plus pour féliciter le traducteur, un galicien du nom de Luis Antonio de Figueroa, «qui a étudié pendant quelques années au Portugal dans un collège de Braga» et a terminé sa carrière universitaire à Valladolid, ce qui en a fait un homme instruit en ces matières.

Les réflexions du censeur éludent l'essentiel, soit le fait qu'il s'agit d'un ouvrage profondément anti-aristotélicien, dont les affirmations sur la question des accidents, du vide, des graves, de la lumière et du feu mettent en cause les doctrines de l'Eglise au profit des découvertes de Newton, avec des nuances caractéristiques des positions péninsulaires dans la querelle des Anciens et des Modernes ( $\left.{ }^{28}\right)$.

La publicité de la Gaceta fait au contraire appel à cet argument:

«podrá servir esta obra para que el buen gusto en la Filosofía se extienda y familiarice con todo género de gentes, para que los que han estudiado la Escolástica y no tienen otros principios, puedan adquirir alguna instruccion en la moderna»

avec toutefois un témoignage d'allégeance à la religion:

* (podrá servir también esta obra) para que haciéndose mas comunes y generales entre los hombres de todos estados y condiciones nas noticias filosoficas de las maravillosas obras de Dios en el cielo, mar y tierra, alabencon mas conocimiento su infinita sabiduría y poder».

(*) V. à ce sujet le brillant essai de Rómulo de Carvalho, A física experimental em Portugal no séc. XVIII, Biblioteca Breve, Instituto de Cultura e Língua Portuguesa, Lisbonne, 1982. 
D'autres ouvrages scientifiques de Teodoro de Almeida sont annoncés dans la Gaceta de Madrid à la même époque $\left.{ }^{27}\right)$, permettant de conclure à l'existence en Espagne d'une demande en ce domaine. La Recreación Filosófica semble pouvoir y répondre, quelque vingt ans après sa publication au Portugal, et rencontre un certain succès, puisque Messeguer, à la mort de Figueroa, demandera licence et privilège d'impression des autres tomes pour lui-même $\left({ }^{28}\right)$. Le décalage chronologique mérite d'être noté, sans que l'on puisse en tirer de significatives conclusions, si ce n'est que cette étude sur les traductions d'ouvrages portugais en espagnol ne prendrait vraisemblablement tout son sens qu'insérée dans une réflexion plus

( $\left.{ }^{27}\right)$ Parmi ces oeuvres, les Cartas físico-matemáticas, publiées à Lisbonne en 1784, soit bien après la chute de Pombal, et donc exclues de cette étude qui s'organise autour du ministre de José I. Je renvoie à leur propos à mon art. cit. «Les souscripteurs espagnols etc.». En 1787 la Gaceta annonce un Compendio de la historia de la Filosofía du P. de Almeida que je n'ai vu mentionné nulle parte, ce qui ne me permet pas de le dater pour ce qui est de son éventuelle publication au Portugal. En 1789 (n. 19), c'est El Filósofo solitario, oeuvre généralement attribuée à $\mathrm{T}$. de Almeida (Lisbonne, 1786) qui est proposé au public. Dans le n..$^{\circ} 19$ de 1790 , encore un ouvrage du P. de Almeida non recensé par Inocêncio: Física experimental, ó instituciones de filosofía natural, dispuestas para el uso de las aulas, y completa instruccion de los aficionados á esta Ciencia que aspiran á saberla con sólidos fundamentos. L'oeuvre, poursuit la réclame, est la version écrite des leçons données à Bayonne et Lisbonne par le P. de Almeida, elle est augmentée d'illustrations et de nouvelles expériences. «Es admirable la claridad y brevedad de este curso de física, en el que nada omite el autor de lo perteneciente a esta Ciencia. Empieza por la mecánica, fundamento de la física, apoyándola en la geometría clarísima de sus Cartas, pasando despues á la hydrostática, astronomía, cosmografia, y a los más ilustres fenomenos de los elementos: en el fuego exâmina las propiedades de la luz y del calor: en el ayre la armonía del sonido ó la música, y la diversidad de ayres que han descubierto los modernos: en el agua los efectos de sus peso para construccion de máquinas, canales ó acequias, los vapores, nubes, fuentes, etc.; pasa despues á los cuerpos compuestos, habla del hombre, de sus sentidos, de la anatomía, con otros puntos que agradarán á los curiosos, y disputa por último de los brutos, de las plantas, del iman, de la electricidad, del trueno y rayo, todo con aquella gracia que se advierte en todas sus obras».

${ }^{(\infty)}$ Il ressort d'un dossier consulté à l'A.H.N.M. Consejos, Leg $^{\circ}$ 5551-5 qu'il y eut procès, à la mort de Messeguer, entre un certain Moncasi, traduteur de Gemidos da Mãe, et de Instituciones físicas, qui avait racheté les droits des héritiers de Messeguer et de ceux de Figueroa, et d'autres prétendants à la traduction et à la publication de T. de Almeida. Ne l'ayant pas encore exploité, je l'évoque cependant comme bonne preuve de l'aspect lucratif offert par ces traductions. 
ample englobant les autres publications annoncées dans la Gaceta, étrangères ou nationales.

Méthode d'étude, traité de la connaissance, les ouvrages de Vernei et de Teodoro de Almeida, dont le propos déclaré est d'instruire sans ennuyer, se prévalent d'une semi-vulgarisation souhaitée et bien reçue du public, un public friand de formules et de conseils, auprès duquel les livres de médecine font recette, car ils sont conçus dans une perspective pratique, concrète, exacte, en accord avec l'esprit du siècle et de ses précurseurs, comme Curvo Semedo, dont la Gaceta de Madrid annonce dans son n..$^{\circ} 21$ de 1763 les Secretos Medicos, y Chirurgicos: traducidos de Lengua Vulgar Portuguesa por el Doctor D. Thomás Cortijo Herraiz, con un Breve Diccionario Lusitanico Castellano, en vente chez Francisco Fernández, calle de las Carretas.

Il semble qu'il faille y voir la reprise d'une publicité antérieure, puisqu' Inocêncio dit posséder un exemplaire de cette oeuvre daté de 1751, ajoutant que le traducteur espagnol s'est servi, selon ses propres dires, de trois ouvrages différents du médecin portugais: Polyanthea medicinal, noticias galenicas, $e$ chymicas repartidas en tres tractados. Dedicados á saudosa memoria do em $^{\text {mo }} \mathrm{sr}$ Cardeal de Sousa, etc. Lisboa, Miguel Deslandes, 1695; Atalaya da vida contra as hostilidades da morte, fortificada e guarnecida com tantos defensores, quantos são os remedios, que no decurso de 58 annos experimentou o doutor, etc. Lisboa, Officina Ferreiriana, 1720; et Observações medicas doctrinaes de cem casos gravissimos, que em serviço da patria, $e$ das nações estranhas escreve em lingua portugueza e latina o doutor etc., etc. Lisboa, Antonio Pedroso Galvão (2. ${ }^{a}$ ed.) 1727. Les trois dates avancées suffisent à elles seules à éclairer le problème de la périodisation des Lumières dans la Péninsule, car l'une d'entre elles est de la fin du XVII ${ }^{e}$ siècle et toutes trois sont largement antérieures à Vernei.

Curvo Semedo semble avoir été le seul médecin portugais traduit en Espagne jusqu'en 1781, où c'est au tour d'un "estrangeirado" que son judaïsme a exilé, Ribeiro Sanches, d'avoir les honneurs de la Gaceta, avec son Tratado de la conservacion de la salud de los pueblos, y consideraciones sobre los terremotos, une traduction par Benito Bails vendue chez Ibarra, du Tractado da conservação da saude dos Povos: obra util e necessaria aos magistrados, capitães generaes, capitães de mar e guerra, prelados, abbadessas, medicos e paes de familias paru à Paris en 1756, et publié à Lisbonne l'année suivante. 
La Gaceta insiste peu sur la nationalité de l'auteur, et beaucoup sur ses titres:

«obra que escribió en Portugués el Doctor D. Antonio Ribeyro Sanchez, primer médico que fue de los Exércitos de Rusia, residente ahora en París».

Elle ne précise pas qu'il était docteur en médecine de l'Université de Salamanque, s'étendant en revanche longuement sur l'oeuvre, qui prétend «fournir les moyens nécessaires pour éviter les maladies des citadins, soldats, marins et navigants». Dans une première partie, on indique "comment purifier et renouveler l'air des maisons particulières, des églises, des prisons et des infirmeries d'hôpitaux et de couvents", et l'on souligne l'intérêt des plantes arômatiques, des bois et des forêts, des vents, ainsi que l'importance du site pour une ville et la nécessité d'eaux de bonne qualité, tous éléments qui concourent à l'existence d'un climat salutaire. La deuxième partie concerne les soldats, en campagne ou en garnison, "auxquels on épargnera les maladies en leur distribuant équitablement besognes et temps de repos" et en leur organisant un régime de nourriture et de boisson approprié. En troisième lieu, l'auteur déclare.

"Como se ha de procurar la pureza del ayre en las embarcaciones, la conservacion de los bastimentos, proponiendo varios modos yá para preservarlos de la corrupcion, yá para corregirla quando se manifieste, y sobre todo la del agua, algunos medios para hacer potable la del mar, y la comida y bebida mas adequada para la sanidad de los navegantes».

(Gazeta de Madrid n.*18)

Ces propos sur l'hygiène militaire prendront une singulière résonance lors des guerres allumées par Napoléon en Europe. On y lit encore de façon plus générale le souci de la santé populaire à une époque où l'essor démographique garantit la force économique d'une nation $\left({ }^{29}\right)$.

$\left({ }^{20}\right)$ On conclut d'une correspondance conservée à l'A.H.N.M. (Consejos, Imprentas y sus agregados, Leg..$^{\circ} 11278$ ), que B. Bails se servit de l'ouvrage de $R$. Sanches pour faire passer ses propres travaux. Il écrit en effet à Floridablanca le 20/12/1790: «me determiné a poner la obra (el Tratado de la conservación) en terminos que nadie pueda echar en ella menos los puntos de Fisica y Quimica enlazados con sus asuntos, de los varios, utiles y estraños descubrimientos hechos desde que Sanchez escribio; transformando con estas mudanzas y adiciones al tratado en otro util y curioso de lo que era antes.» 
L'actualité médicale portugaise est elle aussi présente dans les colonnes de la Gaceta avec l'annonce en 1764 (n. ${ }^{\circ}$ 43) du Diario Universal de Medicina, Cirujia, Pharmacia, etc. Contiene los discursos, y observaciones, trabajados por las dos Academias de Medicina, y Cirugía de Porto, etc. de Manuel Gomes de Lima Bezerra, mais il s'agit de l'oeuvre en portugais, telle qu'elle vient de paraître à Porto. L'académie portopolitaine luso-espagnole créée par Bezerra Lima donnait en effet à ce dernier l'assurance d'un certain nombre de souscripteurs espagnols, puisqu'elle comportait, sous le signe du Zodiaque lusitan - organe de publication de ses productions et symbole de l'institution -, douze cercles de collaborateurs, dont huit espagnols $\left({ }^{30}\right)$.

Ami d'André García Vázquez, chirurgien du Roi d'Espagne, de Francisco de Buendía y Ponce, son médecin, de Juan Luis Roche, diffuseur de la pensée de Feijoo dans la région de Cádiz ( ${ }^{31}$ ), Gomes de Lima peut être considéré comme un esprit curieux et fécond, dont les vues européennes mériteront qu'on s'y arrête un jour plus longuement. Adversaire tenace des doctrines d'António Ferreira, partisan acharné de Boerhaave, il fait triompher ses positions, puisque l'enseignement anatomique dispensé dans l'Université de Coimbra l'est à partir du médecin hollandais $\left({ }^{32}\right)$. Dans le Diario Universal de Medicina, qui semble n'avoir eu que deux numéros, il évoque chaleureusement ses correspondants espangnols avant de publier divers observations, puis des lettres à différents amis, que lui permettent de citer longuement Piquer, son "collègue des Académies d'Espagne, et l'un des plus remarquables commentateurs d'Hippocrate» $\left.{ }^{{ }^{33}}\right)$. Le thème de la lettre en question étant la salubrité de l'air, on n'est pas surpris d'apprendre que Ribeiro Sanches avait envoyé à Gomes de Lima

${ }^{\left({ }^{20}\right)}$ Sur cette curieuse académie, à laquelle je reviendrai nécessairement, consulter l'approche qui en a été faite par António Alberto Banha de Andrade, «Uma Academia Científica Luso-Espanhola, antes da expulsão dos Jesuítas», Brotéria, XL, 1945, pp. 619-636.

$\left({ }^{31}\right)$ Au sujet de J. L. Roche, v. Michel Dubuis, «El erudito Juan Luis Roche, epígono y propagandista de Feijoo en Puerto de Santa María», II Simposio sobre el Padre Feijoo y su siglo, Oviedo, 1981, pp. 285-320.

${ }^{(32)}$ M. Gomes de Lima Bezerra, Memorias Chronologicas e Criticas para a Hist. ${ }^{a}$ da Cirurgia ou Noticias das origens, principais progressos, revoluçoens, descubrimentos, seitas, privilegios, Academias, obras impressas e varoens famosos da Cirurgia desde o principio do Mundo até o presente. Porto, 1779.

(') Ibid. 
son traité sur la conservation etc. et entretint avec lui une correspondance suivie depuis Londres $\left(^{\mathbf{B 4}}\right)$.

Ce Diario, qui était, répétons-le, en version originale, se vendait chez les frères Barthelemi. Faut-il penser que Gomes de Lima s'adressa pour la diffusion de son ouvrage, à l'un des ses confrères madrilènes? Les Barthelemi étaient correspondants de Mayáns et de Cenáculo, lui-même en relation avec Gomes de Lima $\left.{ }^{(35}\right)$. On doit en rester aux conjectures, tout en supposant que la démarche ne devait pas être la même selon qu'il s'agit d'une traduction ou d'une oeuvre en provenance directe de l'étranger.

Un deuxième groupe d'ouvrages portugais traduits en espagnol concerne grosso modo la morale, avec des variantes: religion, moeurs -; ils sont souvent empruntés au passé, comme si l'éthique était en somme une donnée immuable, ou... constamment réadaptable. Vademecum religieux, bréviaires laïques, ils correspondent à des besoins fidèlement récurrents, que scandent pour certains les rééditions.

Ainsi est proposé aux lecteurs de la Gaceta n. 12 de 1754 un livre de conseils moraux, Vindicias de la virtud, y escarmiento de virtuosos, escritas en Portugués por el $R^{m o} P$. M. Fr. Francisco de la Anunciacion, del Ordien de S. Agustin, y traducidas en Castellano por el Doct. D. Fernando de Setien Calderon de la Barca, dont il est précisé qu'il s'agit d'une seconde impression, en deux tomes. L'ouvrage se vend dans la librairie de Francisco Lopez. Il avait été édité en trois fois à Lisbonne, en 1725, 1726 et 1727, sous le litre Vindicias da virtude, e escar. mento de viciosos nos publicos castigos de Hypocritas dados pelo Tribunal do Santo Officio (Officina Ferreiriana). Francisco da Anunciação est mentionné par Barbosa Machado. Né

\section{(4) Ibid.}

( ${ }^{35}$ Ces Barthelemi appartenaient-ils à une famille de libraires dont une branche se serait trouvée à Lisbonne. Un passage d'une lettre de Mayáns semble l'indiquer: «Aviendo venido ayer a visitarme el librero Mallen, le preguntè que no sabia yo en que consistia que los egemplares de las Traducciones Castellanas de Virgilio, que yo avia regalado en Madrid a algunos amigos juntamente con la vida del Poeta que escrivi, avian llegado a manos suyas, i me avian respondido, i del que embie para V. Ex. no tenia yo noticia alguna. Me dijo que èl embio dichos libros para que embiassen por ellos a casa de los hermanos Bartholami, comerciantes de libros, i que le parecia que V. Ex. no avia dado orden para recogerlos, i assi estimarè que V. Ex. mande que los pidan y saquen del poder de dichos Bartholami, porque deseo que V. Ex. dè a conocer estas obras». Valence, 10/12/1778. Ou faut-il comprendre que c'est chez les Barthelemi (my?) de Madrid que Cenáculo devait envoyer un courrier qui recueillît les ouvrages en question? Une lettre de Gomes de Lima à Cenáculo est conservée à la Bibliothèque d'Evora. 
en 1691 à Lamego, il fut professeur de théologie et philosophie et mourut en 1720 .

Du début du siècle également (1716), et annoncé dans la Gaceta n. 37 de 1763, Ultimo instante entre la Vida, y la Muerte, considerado a luz de los desengaños, que el Pecador moribundo concebird, haciendo reflexión sobre su vida pasada, sobre su estado presente, y sobre su suerte futura, "traduit en langue castillane par le Bachelier Francisco Simón Diaz de Colmenár, prêtre» $\left.{ }^{(38}\right)$. On le trouve dans la librairie d'Hypolito Rodriguez, calle de las Carretas.

Sorte de manuel du bien mourir, il a pour auteur le Jésuite Miguel Dias et parut à Lisbonne sous le titre Ultimo instante entre a vida e a morte considerado à luz dos desenganos.

Dans le même genre, un art de prier du $\mathrm{P}^{\mathrm{e}}$ Teodoro de Almeida-on retrouve son nom dans tous les domaines, c'est l'homme-orchestre des Lumières portugaises-,Tesoro de paciencia, o consuelo del alma atribulada en la meditacion del Salvador, proposé aux lecteurs de la Gaceta n. 29 de 1783 sans mention du traducteur, en vente chez Manuel Godos. La demande de licence $\left({ }^{37}\right)$ nous apprend qu'il s'agissait de D. José Francisco Monserrate, qui se chargera aussi du Feliz indepen. dente. Le censeur le félicite pour la justesse de sa traduction. Quant à l'oeuvre, elle est jugée "très utile, car pleine d'une fort saine doctrine, en tous points conforme à notre sainte foi catholique et aux bonnes moeurs". C'est en 1768 qu'avait été publié à Lisbonne ce Thesouro de paciencia nas chagas de Jesus Christo, ou consolação da alma atribulada na meditaçâo das penas do Salvador.

On ne peut négliger à ce sujet l'inscription de ces livres d'oraison ou de méditation dans le courant de la nouvelle rhétorique (dont les Sermones du même Teodoro de Almeida seront un exemple caractéristique dans le dernier quart du siècle). Est-ce à ce titre, et dans le domaine de l'expression oratoire qu'une Oracion traducida del Portugués al Castellano $p^{a}$ visitar la Iglesia los dias de Jubileo est offerte au public dans le n. ${ }^{\circ} 13$ de 1753 de la Gaceta. Elle est en vente chez la Veuve de Diego Barthelemi, sans que nous puissions en déterminer l'auteur ni la date, et accompagne un Tratado util para

(*) A.H.N.M., Consejos, fndice de Impresiones, Leg. 50659. Aravaca accorde la licence le 24 février 1763.

$\left.{ }^{37}\right)$ Ibid. Leg ${ }^{\circ} 5546-112$. Dans la demande de licence figure «en las llagas de Nuestro Señor Jesus-Christo», qui a disparu de la petite annonce. 
meditar, confessar, y comulgar, tiré des Pères de l'Eglise, du Fr. Antonio de Molina.

De quand date cette Carrilla de enfrenar, para Cavalleros aficionados al Arte de montar à cavallo, traducida del Portuguès al Castellano por D. Santiago Romero, vendue dans la librairie de Luis Gutiérrez, calle de la Montera, et annoncée dans la Gaceta n.० 11 de 1751? Barbosa cite divers traités d'équitation, pour la plupart manuscrits d'ailleurs, mais aucun ne porte ce titre.

On va remonter jusqu'au XVII' siècle à nouveau, avec les Avisos para Palacio, de Luís Abreu de Melo, annoncé dans le n. ${ }^{\circ} 41$ de 1761. Ce manuel du parfait courtisan, divisé en chapitres correspondant chacun à l'une des qualités nécessaires à l'homme de cour pour réussir dans ses entreprises, parut à Lisbonne en 1659. Traduit par Eugenio Garsifa, il est vendu à Madrid dans plusieurs librairies, de 1761 à 1774, et aussi dans une joaillerie, au coin de la calle del Pez. Inocêncio mentionne l'édition de 1761 en indiquant que les licences montrent l'existence d'une publication antérieure, datant de $1724\left({ }^{38}\right)$. Une précision qui suscite une certaine perplexité, une fois de plus liée à la périodisation, et me conduit à considérer le problème de ces traductions essentiellement à partir du public, des traducteurs, des censeurs, des libraires. Peu importe, pourrait-on aller jusqu'à dire, la date initiale d'une oeuvre ou ses rééditions si ce n'est comme les marques permanentes de ses possibilités de succès; ce qui compte, c'est de la juger à chaque fois dans son rapport avec l'expectative des lecteurs, telle qu'elle peut être appréciée par ceux qui espèrent en tirer profit, qu'il s'agisse de gains matériels ou de bénéfices idéologiques. On en a pour preuve l'obligation pour le traducteur de déposer pour chaque nouvelle édition une nouvelle demande de licence, qui entraîne un nouveau rapport de censure variant, lui, avec les époques. Ainsi en 1786, Messeguer et Arrufat, le censeur de la Recreacion Filosofica se voit chargé de rapporter sur les Avisos, qui paraissent une nouvelle fois cette année-là accompagnés de la Carta de guia de casados (1651), de Francisco Manuel de Melo. Il émet une opinion doublement favorable:

"Son dos obras diferentes y de distintos autores; y la segunda se volviò a imprimir separadamente en Madrid no hà muchos años. Una y otra están bien

$\left.{ }^{(28}\right)$ Le dossier que nous avons consulté à l'A.H.N.M. est de 1761. La demande de licence est en date du 16 janvier. La censure, remise à D. Leopoldo Gerónimo Puig, de la Royale Académie d'Histoire, manque. La licence est accordée le 4 février. 
escritas, tienen buenas maximas y pueden servir de utilidad»,

ajoutant que la première oeuvre, soit la Carta, est de loin supérieure à la seconde $\left({ }^{39}\right)$. Pourtant quelques années plus tard, ce dernier ouvrage aura droit à un nouveau rapport de censure, en vue d'une réédition, nettement moins élogieux:

aen è se deben suprimir los Parrafos, y clausulas que vàn rayados en los respectivos quadernos, por contener cuentos ridiculos, nada decentes, y opuestos al honor de el matrim ${ }^{\circ}$, y à la seriedad con que debe tratarse materia tan principal de Ntra Sta Religión, y algunas proposiciones equivocas, inductivas à error y mal-sonantes» $\left({ }^{40}\right)$.

Le problème du mariage, de l'entente entre les époux, est au coeur des préoccupations de l'Espagne éclairée. C'est la façon dont il est envisagé d'une décade à l'autre qui donne la clé de la différence entre les rapports de censure pour une même oeuvre, plus que l'oeuvre elle-même qui, on en conviendra, contenait le même nombre d'anecdotes indécentes en 1780 et en 1793.

On peut donc estimer que la distinction objective entre premières éditions et réeditions est à reconsidérer, voire à invalider, puisqu'à partir du moment où il y a demande d'impression, qu'il s'agisse d'un ouvrage ancien ou récent, l'appareil de censure se met systématiquement en marche avec des critères identiques, certes (sauf modification de la législation en vigueur), mais sans cesse modulés selon les exigences de l'idéologie en place et les besoins théoriques du public.

Du fatras de dates considéré en effet, de 1651 à 1768 en ce qui concerne les éditions au Portugal, de 1751 à 1786 pour ce qui est de la parution en Espagne, il serait tentat de dégager une permanence remontant au XVII ${ }^{e}$ siècle, ou pour le moins de signaler une nouvelle fois aux Lumières péninsulaires des prémices bien antérieurs au Teatro crítico et au Verdadeiro Método, et cela, bien qu'Ilustración comme Esclarecimento s'enracinent plutôt dans le XVI ${ }^{e}$ siècle.

Mais il me semble plus éclairant d'avancer une hypothèse sans doute hardie mais néammoins, je l'espère, convaincante.

\footnotetext{
(20) A.H.N.M., Consejos, fndice de Impresiones, Leg. ${ }^{\circ} 50674$.

$\left({ }^{+0}\right)$ Ibid., Leg ${ }^{\circ}$ 5559-18.
} 
Les ouvrages scientifiques recensés plus haut me semblent avoir un point commun avec ceux qui vont de la morale aux moeurs: ils répondent dans leur disparité aux besoins du groupe social qui lit, et qui est avide de méthodes, pour employer un terme global et flou: méthodes d'enseignement et d'apprentissage, méthodes de conservation de la santé et de guérison de la maladie, méthodes de vie pour une société qui s'ouvre et se découvre, méthodes de prière qui assurent la sérénité ici-bas et le salut dans l'au-delà. C'est à cet appétit de concret et de rationnel que répondent, je me risque à l'affirmer, et le Verdadeiro Método de estudar, et le Tratado da conservação da saude, les Segredos de Curvo Semedo et la Guia de casados, L'Ultimo instante et la Carrilla de enfrenar. Ils viennent combler les aspirations d'individus nouveaux, qui se recrutent dans un tiers état en devenir, pour lequel bien monter à cheval, savoir se tenir à la cour, complètent heureusement les savoirs acquis par le travail ou la vertu.

Remarquons que dans cette perspective l'ouvrage de Vernei occupe à la fois l'espace de la philosophie et celui des sciences: presqu'au même moment était publié à Lisbonne un essai philosophique et moral qui clora notre série didactique, Reflexões sobre a vaidade dos homens, ou discursos moraes sobre os effeitos da vaidade, (1752). Cette oeuvre connut de nombreuses rééditions au Portugal, 1761, 1778, avant de paraître avec un appendice intitulé Carta sobre a fortuna en 1786. Est-ce cette Carta qui attira l'attention du traducteur espagnol sur le livre de Matias Aires Ramos da Silva de Eca? C'est elle en tout cas qui est annoncée en premier dans le n. 38 de la Gaceta de Madrid de 1787. Puis, dans le n. . $^{\circ}$ est proposée Reflexión sobre la vanidad de los hombres, version de $\mathrm{D}$. Joseph de Palacio, en ces termes:

«Entre varias reflexiones contiene algunas sobre la vanidad en el amor, en la hermosura, en las mugeres, en la republica de las letras, en la ciencia de hacer justicia, en la sangre, y en la historia de que se sirve la nobleza. Obra muy acreditada que ha corrido con mucha aceptacion en el Reyno de Portugal».

Le livre est en vente chez Gabriel Gómez, calle de las Carretas, $10 \mathrm{rs}$. broché et 16 relié. Les publicités qui le concernent continuent encore en $\mathbf{1 7 9 0 .}$

La censure, favorable, qui fut établie en son endroit ( $\left.{ }^{41}\right)$, comporte diverses remarques qui soulignent encore s'il en était

- (1) Ibid., Leg॰ 55214. 
besoin l'esprit utilitaire qui pnéside à l'autorisation de traités du genre. En effet, si le censeur l'approuve:

«esta Reflexion me parecen unos desengaños muy juiciosos y eficaces para despertar los hombres sepultados en los lisongeros gozos de su vanidad a fin de que aspiren a una perfecta pureza y desinteres en sus dichos, deseos, y acciones (...),

il estime qu'il faut modifier ce lourd et volumineux ensemble, en le divisant en sections ou articles, ou mieux encore en Réflexions, comme son titre l'indique:

«por cuyo medio claro y metodico se clasifiquen todas las especies de vanidad con sus epigrafes respectivos. v.g. vanidad de las sepulturas, vanidad de linaje, vanidad de las letras, vanidad de las armas etc.».

On retrouve là une organisation logique appliquée à l'humain, pareille au déploiement des Avisos, et qui ne diffère pas tant qu'on le croit de la distribution alphabétique des remèdes de Semedo ou de la répartition en lettres du Verdadeiro Método. Le censeur des Reflexões était Antonio de Capmany, alors secnétaire de l'Académie d'Histoire madrilène. Ce grand esprit éclairé, qui avait sans doute gouté les passages consacrés par Matias Aires à l'historiographie et aux dangers de la subjectivité pour son bon usage $\left({ }^{42}\right)$, ne conseillait pas au hasard une présentation maniable, accessible, méthodique en un mot: il se plaçait du côté des lecteurs.

Sa remarque nous permet de cerner mieux encore l'élément qui cimente entre elles les diverses oeuvres portugaises de ce premier groupe retenues par les traducteurs espagnols, accueillies avec bienveillance par les censeurs, et diffusées avec succès dans le public: c'est leur appartenance à une époque en gestation d'abord, en transition ensuite, qu'on a appelée l'âge classique, et dont $\mathbf{M}$. Foucault montre avec clairvoyance et brio le désir d'ordre, de classification, relatif à toutes choses, et surtout peut-être aux inclassables $\left({ }^{43}\right)$.

(2) Sur ce point, v. la subtile étude de L. A. de Oliveira Ramos, «Doutrina acerca da história em escritores portugueses do séc. XVIII: Matias Aires e a História», Bracara Augusta, XXVIII, Fasc. 65-66 (77-78), 1974.

(*) Michel Foucault, Les mots et les choses. Paris, Gallimard, 1966, 400 p., pp. $137-150$. 
Si l'époque a besoin de recettes, si elle apprécie de pouvoir prendre la mesure de l'univers et de l'homme à l'aide de répertoires, glossaires, dictionnaires, etc., elle se cherche également des exemples, dans la réalité et dans la fiction.

Les oeuvres religieuses, édifiantes, auront donc la faveur du public. Que le modèle numéro un soit Jésus-Christ ne nous surprendra pas. C'est à ce titre, n'en doutons pas, que la Gaceta de Madrid n. ${ }^{\circ} 22$ de 1763 annonce à ses lecteurs Trabajos de Jesus, nouvellement traduits des Trabalhos de Jesus publiés à Lisbonne en 1602 et 1609 , et réédités en 1733 . Ce récit est "écrit en Portugais par le vénérable Fr. Thomé de Jesus, de l'Ordre de Saint-Augustin, alors qu'il était captif et prisonnier en Barbarie». On se le procurera auprès du portier de San Felipe el Real. Le traducteur est l'une des grandes figures des Lumières espagnoles, Enrique Flórez, auteur de La España Sagrada, qui polémiqua avec Grégoire Mayáns au sujet de la définition de l'Ere Hispanique, et entretenait des relations épistolaires avec l'érudit portugais Tomás Caetano de Bem, lui-même correspoñdant du grand Valencien. Il demande à plusieurs reprises la licence de réimpression, en $1772\left({ }^{44}\right), 1778\left({ }^{45}\right), 1785\left({ }^{46}\right)$, et elle lui est toujours accordée. L'oeuvre avait été traduite en espagnol pour la première fois en 1624, par Juan de Lanaya.

La Gaceta n..$^{\circ} 2$ de 1760 proposait, elle "un livre nouveau, en deux tomes: La Vida del Gran Padre, y Patriarca San Phelipe de Neri: escrita en Portuguès por el Padre Don Manuel Consciencia, Presbytero de la Congregacion de Lisboa». Il est traduit par un religieux de la Congrégation de Baeza et vendu aux portes de l'Oratoire de Saint Philippe Neri, plazuela del Angel. L'ouvrage était paru à Lisbonne en 1738 , sous le titre Vida admiravel do glorioso taumaturgo de Roma... S. Filippe Nery. L'Oratoire devait connaitre la faveur de Pombal, puis son inimitié, précisément en cette année $1760{ }^{\left({ }^{47}\right)}$ qui voit triompher en Espagne la biographie de son fondateur $\left({ }^{48}\right)$.

(*) A.H.N.M. Consejos, fndice de Impresiones, Leg ${ }^{\circ} 50666$.

(*5) Ibid., Leg 50672 .

(“) Ibid., Leg 50673.

(") Sur la polémique de l'Oratoire avec Pombal, v. A. A. Banha de Andrade, «Processo pombalino contra os Oratorianos», Arquivos do Centro Cultural Português, 1969, pp. 270-296.

(') La censure est d'Aravaca qui pense que al'oeuvre contribuera beaucoup à l'édification des fidèles» (A.H.N.M. Consejos, fndice de Impresiones, Leg $^{\circ}$ 50654.) Le dossier montre une rivalité entre l'Oratoire de Baeza, qui avait le premier reçu l'autorisation de publier l'ouvrage, et celui de Madrid. C'est ce dernier qui l'emporte, la licence étant retirée à Baeza. Nouvel indice de l'intérêt qu'il y avait à se réserver des droits de publication. 
A côté de ces vies réelles, exemplaires, dont on ne saurait négliger l'aspect merveilleux, suscité en particulier par la relation des miracles (nombreuses sont les vies de saints qui se disputent les colonnes de la Gaceta), il est intéressant de voir apparaître des livres de caractère fictionnel. Précurseurs de l'esprit nouveau, ces premiers balbutiements romanesques préparent à n'en pas douter l'extraordinaire succès en Espagne du Feliz independente du P. Teodoro de Almeida, postérieur aux limites chronologiques que je me suis données ici. Le premier en date de ces ouvrages est Alivio de Tristes, y consuelo de Quejosos, annoncé dans la Gaceta n. 39 de 1762: "écrit en Portugais par le P.e Matheo Ribeyro, traduit en castillan par le Docteur Juan Antonio Mora, on le trouvera aux Portes de San Yuste, sur les marches de San Phelipe, et à l'entrée de la calle de las Postas». Cet imposant "roman» était paru à Lisbonne entre 1672 et 1687, et avait été réédité en 1737 et 1764 . Inocêncio avoue avoir pris grand plaisir à cet ensemble de nouvelles reliées les unes aux autres par un fil aussi ténu qu'artificiel, et dit l'avoir relu maintes et maintes fois à l'âge de huit-neuf ans, ému qu'il était par la féconde imagination et la vivacité d'esprit de son auteur. On pourrait en effet détacher de l'oeuvre l'histoire de Lisardo et Theodora, celle de Sancho et Lisarda, celle de Roberto et Eugenia, etc. A chaque fois, et après avoir absorbé l'indigeste salmigondis de citations latines et de références historiques dont est farci le récit à des fins prétendûment moralisatrices, on est appelé à se repaitre d'aventures amoureuses peu recommandables, où l'inceste et le rapt sont pain quotidien.

Dans le même esprit sont offertes au public esnagnol les Novelas de M. Marmontel y de la Madre Sor Marina Clemencia. traducidas del Francés y Portugués al castellano (Gaceta n. ${ }^{\circ} 48$ de 1774)). Traduites par Antonio Ruiz y Minondo, elles sont distribuées au public par la librairie Escrivano, calle de Atocha. Connue sous le nom de Maria do Céu, auteur en particulier de A Fénix apparecida (1716), Triumphos do Rosario (1740), Enganos do bosque (1741), on peut la considérer comme l'une des premières "romancières" portugaises, créatrice d'intrigues amoureuses aux résonances psychologiques ténues mais délibérément suggérées. Mais il faudrait avoir un exemplaire du recueil proposé pour savoir laquelle de ses oeuvres fut élue. Un rapport de censure favorable concernant une Fénix apparecida et émanant du vicaire erclésiastique ne peut être retenu car il est de septembre $1785{ }^{\left({ }^{49}\right)}$. On notera cepen-

${ }^{\left({ }^{\circ}\right)}$ A.H.N.H. Consejos, fndice de Impresiones, Leg $^{\circ}$ 5551-12. 
dant au passage que le traducteur qui demande la licence... est une traductrice, D. ${ }^{a}$ Maria Antonia Varela, comme si cette oeuvre de femme dût trouver une interprète du même sexe, ce qui n'est pas sans intérêt dans un siècle qui pousse les beaux esprits en jupons à se produire et à s'émanciper $\left({ }^{50}\right)$.

Ainsi une littérature romanesque, ici féminine et précieuse, alimente les rêveries de ces lecteurs et lectrices espagnols qui touchent le dernier quart du siècle.

Est-ce à dire que ce même public épris de leçons aimerait faire l'école buissonnière? Ou qu'une diversification du public, si elle n'existe, est recherchée? On ne s'interdira par de penser qu'une époque que la raison subjugue cherche des échappatoires, l'éclosion du romantisme s'expliquant en partie par la rupture entre un monde que l'on croit pouvoir quantifier et l'infini tentateur de l'irrationnel, en un mot du sentiment.

En tout cas, des élans de la piété à ceux du coeur, il n'y a pas tant d'écart qu'on l'imagine, si l'on se place dans l'optique d'une littérature d'évasion, aussi inclurai-je dans cette seconde série un ouvrage "prophétique», la História do Futuro du P. Vieira, dont la traduction est annoncée dans la Gaceta n. 33 de 1762: Historia de lo Futuro, Prologomeno a toda la Historia de lo Futuro, en que se declara el fin, y se prueban los fundamentos de ella, en vente "dans la librairie d'Antonio Sanz, calle de la Paz». Remarquons au passage que la mention Livro anteprimeyro de l'édition-princeps portugaise (1718) a disparu, peut-être à cause de sa relative incongruité. Ce qui compte, c'est que cet ouvrage messianique et millénariste trouve sa pla-

$\left(^{50}\right)$ Chose curieuse, un Narciso Varela demande également l'autorisation d'imprimer un oeuvre qu'il affirme traduite de Teodoro de Almeida, La Preciosa. Elle est en effet annoncée dans la Gaceta de Madrid n. ${ }^{\circ} 23$ de 1792, avec la précision «alegoría moral». Inocêncio ne recense pas cet écrit de l'oratorien. La version espagnole se trouvant à la Bibliothèque Nationale de Madrid, je l'ai consultée en relevant par acquit de conscience noms des personnages et enchaînement des chapitres. Quelle n'a pas été ma surprise de trouver $A$ Preciosa parmi les oeuvres de Maria do Céu, et de constater en la parcourant que La Preciosa espagnole et prétendûment traduite du P. de Almeida n'était qu'une version abrégée - reste à savoir comment - de la fiction allégorique publiée à Lisbonne en 1731 et 1733. La demande de licence (1787. A.H.N.M. Consejos, indice de Impresiones, Lego 5553-40), comporte à la fois le nom de $\mathrm{T}$. de Almeida et celui de la Mère Marina Clemencia, ce dernier disparaissant et de la réclame et de l'ouvrage. T. de Almeida était fort en vogue en Espagne, se vendait bien: le traducteur a dû penser qu'il aurait plus de succès pour sa Preciosa en l'attribuant à un auteur connu! 
ce dans la sensibilité espagnole, comme le soulignait avec finesse Hernâni Cidade en 1953:

"O Céu em ambos os países peninsulares estava muito perto da terra, interessado pelos destinos desta, porque neles se realizavam desígnios seus. Interrogavam-se os profetas maiores e menores, os bíbli. cos e os de casa; interrogavam-se os astros, com seriedade e aparato quase científicos, segundo regras que na Península foram codificadas, justificadas em livros lidos e acatados, que circularam e se publicaram até muito dentro do sézulo XVIII; interpretavam-se prodígios e prognósticos que se multiplicavam em termos que tornavam o milagre e o maravilhoso comum e quotidiana forma de intervenção dos poderes transcendentes nas misérias humanas» $\left({ }^{51}\right)$.

Une lettre conservée à Simancas, adressée à l'Inquisiteur Général le 9 juin 1777, et dont l'auteur semble être Manuel de Roda, Secrétaire de Grâce et de Justice, va dans le sens que je préconise. Les Inquisiteurs souhaitent certaines suppressions dans l'édition des Sermons de Vieira de 1664, suppressions qui figureront dans l'index de 1790. Voici l'opinion de Roda:

"La expurgacion de estas solas expresiones es una (...) aprobasion de todo lo demas, siendo asi, aue todos los Sermones del P. ${ }^{e}$ Vieira estàn llenos de dis. cursos sutiles, y extravagantes, torciendo y violen. tando el sentido de la sagrada escritura contra la comun $\mathrm{y}$ verdadera inteligencia de los Santos $\mathrm{Pa}$ dres, y de la universal Iglesia, en que contraviene al expreso decreto del concilio de Trento sesion A. Es inmenso el daño que ha causado este Autor ron cl aplauso que han logrado sus Sermones por haver querido imitarle en su desarreglada fantasia muchissimos Predicadores, y asi debian de (...) prohibirse asi sus sermones como la historia de lo futuro y la defensa de ella que se halla en su obra posthuma en apoyo del error de los minelarios." $\left({ }^{52}\right)$.

(") P. António Vieira, Obras escolhidas, VIII, História do Futuro, Introduction. Sá da Costa. Lisbonne, 1953.

(2) A.G.S. Gracia y Justicia, Leg. ${ }^{\circ}$ 627. C'est moi qui souligne. 
Pour apporter de l'eau à mon moulin, j'utiliserai un rapport de censure concernant une dernière oeuvre, pieuse, du P. Teodoro de Almeida, publiée à Porto en 1763: Gemidos da Mãe de Deus afflicta, ou estimulos de compaixão das suas do. res (Ed. Francisco Mendes Lima) et proposée aux lecteurs de la Gaceta de Madrid n. 14 de 1790 sous le titre Gemidos de la Madre de Dios $\left({ }^{53}\right)$. Traduit par Joseph Moncasi, il est vendu chez Castillo, Esparza et Copin. Les attendus du censeur, qui condamne l'oeuvre - alors qu'en général T. de Almeida est particulièrement apprécié par le despotisme éclairé espag. nol -, sont en effet fort éloquents:

«nosotros abundamos en este genero de devocionarios a la Virgen S. ${ }^{\text {ma }}$ (especialmente en sus dolores) tan piadosos como los del P.e Almeida y mas nutritivos de la verdadera piedad. Si por esta razon (...) es superflua la impresion de esta trad. ${ }^{\text {on }}$, por otras la juzgo en cierto modo, peligrosa, todo el empeño del P. Almeida es hacer llorar a los devotos de la S. ${ }^{\text {ma }}$ Virgen, precisam. ${ }^{\text {te }}$ por $\mathrm{q}^{*}$ la Sr. ${ }^{\text {a }}$ sintò y lloró durante la vida, y pasion de su unigenito hijo. Esto es trastornar las cosas y sacarlas de su centro (...) La Iglesia nos excita en sus versos, no a llorar por

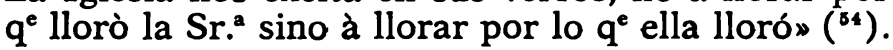

Cette émotion pour l'émotion, ces larmes, pour les larmes nées de la compassion du lecteur - de la lectrice? évoquant la Vierge douloureuse sans avoir assez présent à l'esprit de calvaire du Christ, sont condamnables, comme l'est, poursuit le censeur, d'avoir montré une Vierge exagérément plaintive, et par là même départie de sa dignité, "exemple répréhensible et propre à séduire les âmes simples de l'autre sexe (sic)». Enfin l'oeuvre abonde en "cas prodigieux" non prouvés, qui font du $\mathrm{P}:$ de Almeida l'une de ces «âmes simples, qui croient tout ce qui parait miracle sans autre examen que leur pieux sentiment» $\left({ }^{55}\right)$.

Mysticisme diffus, épanchements intimes, merveilleux, tous ingrédients que cette décadent, larmoyante, fin de siècle est prête à absorber, de préférence aux nourritures plus frugales qu'un rationalisme accablant lui confectionne sans trêve. Mais l'actualité, dira-t-on? L'actualité du Portugal pom-

(\$) La demande de licence est de 1788. A.H.N.M. Consejos, fndice de Impresiones, Leg $^{\circ}$ 5554-98.

(s) Ibidem.

(s) Ibidem. 
balin? Se fraie-t-elle un chemin entre une morale en évolution et une science en révolution?

Elle est présente en effet, à un moment stratégique: l'expulsion de Jésuites hors d'Espagne. C'est en 1768 et 1769 qu'apparáissent dans les colonnes de la Gaceta des oeuvres portugaisës qui s'inscrivent dans les deux grands courants de la politique de Sebastião de Carvalho: le "régalisme» et l'anti-jésuitisme, en une symbiose caractéristique du régime. Le premier ne fait qu'affleurer tout d'abord, avec la Respuesta apologética de Antonio Pereyra, Presbytero de la Congregacion del Oratorio de Lisb.sa al Padre Gabriel Galindo, Theologo de Madrid, o a la Censura que éste hizo de su Tentativa Theologica, impresa en Lisboa, sobre el poder de los Obispos en tiempo de Rotura con la Corte de Roma, annoncée sans nom de traducteur dans le $\mathrm{n}^{\circ} 33$ de 1768 . Il ne fait qu'affleurer parce que la Tentativa Theologica n'a pas été, elle, traduite. Cependant, elle circule en version originale dans toute l'Espagne, avec d'ailleurs l'autorisation officielle de sa lecture, puisque le censeur Juan de Aravaca ne voit pas de raison pour s'opposer à la diffusion de l'ouvrage du Febronius portugais quand on lui demande son avis après saisie du livre en douane:

"La Tentativa Theologica del Dr. Antonio Pereyra, en que se pretende mostrar que impedido el recurso a la Sede Apostolica, se debuelve a los Obispos la facultad de dispensar en los impedimentos publicos del Matrimonio, y demas casos reservados al Papa; como es materia de Disciplina, y el Autor lo propone por via de Question, y reducida al caso de no haber otro recurso para proveher al govierno de las Iglesias: no hallo inconveniente en que se introduzca en el Reyno este Libro, que està adornado de erudicion selecta».

$$
(14 / 8 / 1767)\left({ }^{50}\right)
$$

Nicolás de Azara, ambassadeur d'Espagne à Rome et à Paris, considérait également qu'il s'azissait d'une lecture à conseiller:

"Si se llegasen a divulgar dos o tres buenos libros no más, sobre estas materias, en un año se ilustraría España $\mathrm{p}^{\mathrm{a}}$ siempre: Febroniọ, Pereira no son ma-

${ }^{(6)}$ A.H.N.M. Consejos, fndice de Impresiones, Leg $^{\circ} 50685$. 


\section{Espagne des Lumières}

los, a falta de otros mejores. Bossuet y. Fleury son mejores» $\left({ }^{57}\right)$.

Pereira de Figueiredo avait adressé la Tentativa, publiée à Lisbonne en 1766, au P. Gabriel Galindo, qui lui avait fait tenir sa réponse critique par une lettre du 17 juillet 1767 . Cette Censura précédait la Resposta apologética dans la brochure publiée peu après au Portugal. Il en allait de même dans la traduction madrilène, qui permettait donc aux lecteurs espagnols, auxquels la librairie Joseph Batanero, l'avait fournie, non seulement de se faire une idée claire du débat, mais encore de prendre connaissance par déduction, à partir des problèmes soulevés et de leur résolution, de la Tentativa elle-même. Les positions gallicanistes de son auteur, sa mise en doute de l'infaillibilité du pape, son anti-thomisme, se trouvaient ainsi répandues par cet habile biais. Le Roi ne s'y trompa pas, exilant Galindo, dit Inocêncio, «à quarante lieues de la Cour». Plus tard, une version simplifiée de la Tentativa, mise au point par un certain Cestari, circula, et l'on sait que le ministre de Carlos IV Mariano Urquijo souhaitait voir traduit l'ouvrage de Figueiredo $\left({ }^{58}\right)$. Inocêncio pensait en 1800 qu'il allait l'être incessamment, mais, s'il passe en effet dans sa version espagnole par les mains de divers censeurs tout au long de l'année 1799 , il semble ne jamais avoir vu le jour $\left({ }^{50}\right)$. C'est donc la Respuesta apologética qui véhicula un des aspects" essentiels de la politique pombaline, s'exprimant sous la plume d'un de ses plus actifs et plus fidèles coryphées.

En 1769 , c'est tout l'arsenal anti-jésuitique mis en oeuvre au Portugal au plan théorique depuis l'expulsion de la Compagnie dix ans auparavant qui prend position dans les colonnes de la Gaceta.

A tout seigneur tout honneur: l'épais réquisitoire contre les Jésuites, probablement rédigé par Pombal lui-même, la Dedução Cronológica e Analítica, publiée à Lisbonne en 1767,

${ }^{\left({ }^{57}\right)}$ Cit. par Rafael Olaechea, Las relaciones hispano-romanas en la segunda mitad del siglo XVIII, Zaragoza 1965,2 vol. p. 402 Sur l'intéressante figure du Caballero de Azara, ibid., en particulier pp. 337-362.

$\left({ }^{58}\right) \cdot$ Id., ibid., p. 539.

(ㅇ.) Un gros dossier est conservé à l'A.H.N.H. (Estado 3014), que je n'ai pu encore exploiter. Le traduteur, Caseda y Muro, y défend l'ouvrage de Pereira ec un livre de Cestari, intitulé El espiritu de la jurisdiccion eclesiastica sobre la consagracion de los obispos, qu'il a également traduit: 
est annoncé le premier de la façon suivante dans le $n .^{\circ} 3$ de 1769:

"Deduccion Chronologica y Analitica, en que se manifiestan los horrorosos estragos causados por la Compañia llamada de Jesus, en Portugal, desde que entró en este Reyno el año de 1540, hasta su expulsion en el de 1759: escrita por el doctor Saabra da Silva, Ministro de la Casa de la Suplicacion y Procurador de la Corona; traducida del Idioma Portugués por el Doctor D. Joseph Maymó y Ribes, Abogado del Colegio de esta Corte, en tres tomos, ilustrada con Notas; Trata asimismo el autor en esta Obra muchos puntos de Derecho público, de la suprema independiente Potestad de los Principes en lo temporal; de la del Papa solo en lo espiritual. De la Bula de la Cena y su repulsa en todas las Cortes de Europa; del origen de las Censuras, u remedios de que licitamente han usado y usan los Principes Soberanos quando se abusa de ellas: de la impresion y prohibicion de Libros a quien compete, etc."

On le voit, la publicité était bien faite, et n'y allait pas par quatre chemins. Pourtant Maymó, le traducteur de Vernei, ne semble pas avoir encouru les foudres royales, sans doute parce que la Dedução se plaçait nettement sur un terrain portugais, et qu'il était donc moins difficile de fermer les yeux. D'autant que la doctrine exposée convenait fort à Carlos III.

Dans le n. 48 de la même année est proposée au public une compilation de textes et documents relatifs aux méfaits de la Compagnie, principalement au Paraguay: Causa Jesuitica de Portugal, ò Documentos autenticos, Bulas, Leyes Reales, Des. pachos de la Secretaria de Estado, y otras piezas originales, que precedieron a la reforma, y motivaron despues la expulsion de los Jesuitas de los Dominios de Portugal, en que se halla la República del Paraguay y Marañón, que contiene la relacion de la guerra que sustentaron los Jesicitas contra las Tropas Españolas y Portuguesas en el Uruguay y Paraná: traducidas del Latín y Portugués, e ilustradas, en esta edicion Española». C'est la librairie Francisco Fernández, déjà distributrice de la Deduccion, qui diffuse l'ouvrage.

Un exemplaire en est conservé à la Bibliothèque Nationale de Lisbonne; il offre un prologue révélateur, dans lequel 
sont soigneusement mis en parallèle par l'éditeur les situations portugaise et espagnole jusqu'à la conclusion:

"Como son comunes estos hechos á ambas Naciones, y ponen en claro las ideas ambiciosas de la Compañia, incompatibles con toda Monarquía bien gobernada, no podrá dexar de ser grata al Público la lectura de unos Documentos que desenvuelven por ventura el mayor misterio de la Cábala Jesuítica».

C'est enfin une affaire plus spécifiquement portugaise, mais d'indéniable résonance péninsulaire, qui se donne à connaître par l'intermédiaire de la Gaceta, celle des Jacobéens $\left({ }^{80}\right)$. Rappelons que cette secte, créée à la faveur d'un courant religieux né chez les Ermites de Saint-Augustin, était favorable à la subordination du temporel au spirituel. Elle avait trouvé un soutien inattendu auprès de l'évêque de Coimbra, D. Miguel da Anunciacão, sous la forme d'un mandement manuscrit diffusé auprès des hérétiques qui interdisait la lecture de Dupin et Febronius, auteurs de prédilection de Pombal. D. Miguel prenait donc partie pour le Pape au moment même de la rupture des relations entre Lisbonne et la Curie. Il fut jeté en prison, aczusí avec les autres Jacobéens de sigillisme, c'est-à-dire de violation du secret de la confession. Bien que cette dernière critique semble mettre en rapport les pratiques jacobéennes avec celles des calvinistes, les sigillistes furent assimilés aux Jésuites, en particulier dans la sentence prononcée par la Real Mesa Censória à l'encontre de la Pastorale de D. Miguel, qui est précisément la pièce annon ośe dan la Gaceta n. 20 de 1769: Sentencia de la Real Mesa Censoria contra la Pastoral manuscrita, que el Obispo de Coimbra D. Miguel de la Annunciacion esparció clanciestinamente entre los Párrocos de su Diocesis: proferida en Lisboa en 23 de Diciembre de 1768 ( $\left.{ }^{61}\right)$ : o Apendice a la Deduccion Chronologica, y Analitica. Précisons que la Sentencia, présentée en effet comme "appendice» à la Deduccion est incluse dans l'édition de la Respuesta apologética que j'ai consultée à Lisbonne (Madrid, en la Imprenta de la Vda de Elíseo Sánchez, 1768). La Gaceta indiquait à ses lecteurs la librairie

(c) Sur cette affaire, v. Jacques Marcadé, Frei Manuel do Cenáculo Vilas-Boas, évêque de Beja, archevêque d'Evora, 1770-1814, Centre Culturel Portugais, Paris, 1978, 592 p., pp. 55-59.

(1) Seniença da Real Mesa Censória sobre a Pastoral manuscrita etc., Collecção das leys promulgadas e sentenças proferidas nos casos da infame pastoral etc. Lisboa. Régia Officina Typographica, 1769. 
Alfonso de la Higuera. L'impression Sánchez y ajoute celle de Joseph Batanero.

Les années 1768-1769 de la Gaceta de Madrid reflètent donc très clairement l'offensive contre la Compagnie menée par le pouvoir, et il faudrait voir si les autres ouvrages annoncés, quelle que soit leur provenance, sont orientés en ce sens. Les décrets pris par les différents parlements français (Paris, Aix, Rouen) à l'encontre des Jésuites sont en tout cas reproduits à la fin de plusieurs des numéros de 1767 .

En 1768, outre que sont proposées au public les Apologias de Maymó, qui jouent un rôle identique au plan doctrinal, on peut signaler les réclames faite à deux traductions de type triangulaire sur le même thème: la première est la version espagnole de Reflexões de um Portuguez sobre o Memorial apresentado pelos Padres Jesuitas á Sanctidade do Papa Clemente XIII, expostas em uma carta na lingua italiana a um amigo em Roma, e traduzidas fielmente na portugueza s. l. s. nom d'imprimeur, $1759\left({ }^{82}\right)$, offerte aux lecteurs de la Gaceta n. 30 , avec omission d'ailleurs des mots de um ingenio português qui figure dans le titre. La seconde concerne Instrucção a Principes, sobre a politica dos Padres Jesuitas ilustrada com largas notas e traduzido do italiano em portuguez, Lisbonne, sans nom d'imprimeur, $1760\left({ }^{63}\right)$; dans la petite annonce de la Gaceta $n .^{\circ} 36$, elle est accompagnée du célèbre «Suplemento de la Religion Orthodoxa de los Jesuitas». La traduction del'Appendix às reflexões do Portuguez sobre o Memorial do P. Geral dos Jesuitas, apresentado á sanctidade de Clemente XIII, ou seja resposta do amigo de Roma ao de Lisboa. Impressa em Genova, e traduzida em portuguez. s. 1. s. nom d'imprimeur 1759, autre triangulaire, apparaît également dans la Gaceta, en 1769, dans le n. $^{\circ} 8$. Il s'agit donc véritablement d'un tir groupé, dont il est intéressant de noter que, selon Inocêncio, c'était Pombal lui-même par l'intermédiaire de l'imprimeur Pagliarini, médiateur des intérêts portugais à Rome, qui suscitait ce type de publications.

( $\left.{ }^{82}\right)$ Un mss, de ces Reflexiones de un ingenio português sobre el Memorial presentado por los Padres Jesuitas a la Santidad de Clemente XIII año 1759 se trouve à la B.N.M., ainsi qu'une copie du Memorial presentado por el General de la Compañia de Jesús a Su Santidad Clemente XIII en 31 de julio de 1759.

${ }^{\left({ }^{e 3}\right)}$ Selon Inoc ncio la première impression daterait de 1617 à Milan. L'édition italienne qui donna lieu à la version portugaise comportait le frontispice suivant: Noemenia tuba maxime clangens, sicut olim clanxerunt unisonae prima et secunda tuba magna Lusitana buccinante ad principes universos. Typis mandata Ulyssis. Augustae 1759. Apud Haeredes Bonae Fidei et consocios. Il y avait une dédicace au comte d'Oeiras. 
Ce qu'il faut souligner, c'est que ces deux années, sur l'ensemble de la période étudiée, sont les seules où la littérar ture politique portugaise trouve place dans les colonnes de la Gaceta: années importantes au Portugal, où Pombal assoit théoriquement la mesure d'expulsion prise à l'égard de la Compagnie et la rupture avec la Curie, années brûlantes en Espagne où l'on commence après s'être débarrassé des Jésuites, à poser le problème de l'«épiscopalisme» ou en tout cas de la légitimité du pouvoir temporel pontifical.

L'étude exhaustive que j'ai menée, sur un corpus de vingt-deux traductions en espagnol $\left({ }^{04}\right)$, trois traductions en provenance de l'italien, deux ouvrages en version originale $\left({ }^{05}\right)$, dont un en latin, soit vingt-sept publications $\left({ }^{88}\right)$ au total, dont une moitié seulement est postérieure à 1750 , permet d'ébaucher un certain nombre de conclusions ouvertes.

D'abord, que cette poignée de titres considérés à partir de l'époque pombaline représente probablement malgné sa minceur un bon échantillonnage de l'ensemble des traductions, si ce n'est de la production littéraire, proposés par la Gaceta de Madrid à ses lecteurs: la période obtenue couvrant quarante années de vie intellectuelle espagnole peut coïncider avec une génération, et d'autre part, à peu de choses près, elle embrasse le règne de Carlos III, représentant indéniable du despotisme éclairé en Espagne.

( ${ }^{(4)}$ Je n'ai pas comptabilisé la Carta de Guia de Casados, qui a servi mon argumentation sur la validité du critère de réédition, parce que l'oeuvre est annoncée en dehors de la période pombaline et publiée un siècle avant. Même chose pour la Carta sobre la fortuna, de M. Aires, parue, elle, en 1786 à Lisbonne.

${ }^{\left({ }^{65}\right)} \mathrm{J}$ 'ai laissé de côté la troisième oeuvre, Historia Universal dos terremotos que tem havido no mundo, de que ha noticia desde a sua creação até o presente, Lisbonne, Antonio Vicente da Silva, 1758, de Joachim Joseph Moreira de Mendoza pour la bonne raison qu'elle n'est proposée au public espagnol que dans la Gaceta n. ${ }^{\circ} 39$ de 1783 lors d'un nouveau tremblement de terre à Messine. On ne peut donc la faire entrer sans artifice dans la polémique scientifique sur les séismes, et elle relève davantage d'une actualité de circonstance qui la réactive.

${ }^{\left({ }^{\circ}\right)}$ Naturellement le Portugal est présent sous une autre forme dans la Gaceta par les nouvelles qui en sont données, par exemple lors de la guerre de 1762 pendant laquelle surgissent des traités géographiques espagnols relatifs au Portugal... En dehors de la période choisie, il faudrait évoquer les productions de circonstance liées au mariage des infants d'Espagne et de Portugal en 1785. A partir de 1787, les oeuvres de Teodoro de Almeida criblent la Gaceta de petites annonces détaillées.. Il faut d'ailleurs dire que le «pilonnage» des lecteurs, c'est-à-dire la réitération des publicités, va en augmentant, et qu'il est intéressant de les recenser également pour rendre compte avec plus de précision encore de l'«impact» du Portugal dans les colonnes du journal madrilène. 
Ensuite, qu'il faut les appréhender selon une double optique. L'optique portugaise tout d'abord, et l'on retiendra dès lors essentiellement le passage dans le domaine public espagnol de quelques grandes manifestations culturelles et idéologiques de l'Esclarecimento, cristallisées autour du Verdadeiro Método, la Recreação Filosofica, la Tentativa Theologica, la Deducção Chronologica, le Tractado sobre a conservação da saude dos povos et les Reflexões sobre a vaidade dos homens. Les grands noms qui leur sont attachés ont un point commun: leur relation à Pombal, de l'entente privilégiée, comme pour Pereira de Figueiredo à l'inimitié: comme pour Teodoro de Almeida, en passant par le respect mutuel, ce qui est le cas de Vernei ou Ribeiro Sanches. Le ministre de José I cst-il pour quelque chose dans le fait que les ouvrages du $\mathrm{P}^{\cdot}{ }^{e}$ de Almeida attendent la fin du siècle pour paraître en Espagne? C'est probable, car c'est lorsque l'oratorien publie au Portugal, après la chute du favori, des oeuvres à succès comme $O$ feliz independente, qu'on "exhume» ses travaux antérieurs. Ce qui est sûr en tout état de cause. c'est que la doctrine de Sebastião de Carvalho se déploie dans toute son ampleur autour de l'expulsion des Jésuites. On constatera don= objectivement que les idées portugaises, novatrices voire subversives, franchissent la frontière, et on s'en félicitera.

Toutefois il semble bien aue le regard doive être jeté sur cette production en partant d'un point de vue espagnol. En effet, si ces oeuvres sont retenucs en Espagne, c'est en particulier parce qu'elles répondent et correspondent à n'en nas douter aux besoins d'un public en augmentation, issu d'un groupe social montant qui veut étayer scn ascension sur une scienze, un comportement. une morale, une religion adaptés à la nouveauté de ses principes. J'ai notié au passage un certain décalage chronologique qu'il serait léger de mettre seulement au compte d'un retard de l'Espagne par rapnort au Portugal et peut tenir à des raisons circonstancielles, de politique mais aussi tout simplement d'édition.

Faut-il restreindre la portée de cette pénétration des idiées éclairées portugaises en Espaọne au regard des grands courants de pensée qui traversent l'Europe et se manifestent entre autres par la majorité écrasante de traductions du français annoncées par la Gaceta? Il me semble au contraire que la présence notable des traductions portugaises souligne la spécifité des Lumières ibériques, qui offrent entre elles une étroite parenté, que la fréquence de rapports de censure favorables illustre opportunément.

La distinction que j'ai cru bon d'établir entre littérature méthodologique, littérature politique, et littérature d'évasion 
prête à discussion, car elle est de toute évidence réductrice, et implique d'inévitables chevauchements surtout dus à la latence de l'élément religieux dans toutes les productions. Elle me paraît cependant rendre compte des goûts du public, du choix des censeurs, de l'ambition des traducteurs. En ce qui concerne ces derniers, il serait certainement intéressant d'en dresser une liste précise et d'en étudier le profil. A côté d'exemples fameux comme Enrique Flórez, ou d'importance, comme Maymó et Bails, il n'est pas impossible que l'on voie se dégager en fin de période des entreprises plus professionnalisées, à tournure monopolistique, se disputant les auteurs à succès à des fins lucratives, la perspective de l'intérêt ne devant d'ailleurs jamais, même au départ, être naivement écartée au profit de prétendus élans généreux et gratuits.

Quant à la censure en Espagne, si elle connaît une ćvolution que la lógislation accompagne, elle dépend aussi, bien entendu, de l'orientation des gouvernants, et de la personnalité des censeurs ou des intervenants en ce domaine, dont on retrouve continuellement les noms: Curiel, Aravaca, Messeguer y Arrufat par exemple, et sur lesquels on pourrait s'attarder.

La clientèle du livre enfin semble se diversifier, comme celle de la presse, à partir d'une plus grande expansion de la lecture, et il faut donc plaire à un éventail social de moins en moins étroit qui réhabilite le sentiment aux dépens de la raison $\left({ }^{67}\right)$.

Pourtant la littérature méthodologique et la littérature d'évasion restent en contrepoint l'une de l'autre tout an long de la période, comme si l'on pouvait arriver à une distribution bi-partite, entre les écrits qui s'enracinent dans le réel, ce qui comprendrait alors également le politique, et ceux qui basculent dans l'irréel. Quelle est alors l'incidence de l'actualité? N'est-il pas curieux de voir annoncés la Historia de lo futuro et Alivio de tristes au moment de la guerre de 1762?

Je laisserai ces questions en suspens, qui supposent une étude plus globale et plus approfondie dépassant largement la seule zone d'influence portugaise.

(") Les points de vente sont nombreux et concurrentiels. Pour ce qui est des coûts, ils sont comparables à ceux des ouvrages espagnols. V. mon art. cit. sur \&Les souscripteurs espagnols etc.». 